\title{
Abolishing cAMP sensitivity in HCN2 pacemaker channels induces generalized seizures
}

\author{
Verena Hammelmann, ${ }^{1}$ Marc Sebastian Stieglitz, ${ }^{1}$ Henrik Hülle, ${ }^{1}$ Karim Le Meur, ${ }^{1}$ Jennifer Kass, ${ }^{1}$ \\ Manuela Brümmer, ${ }^{1}$ Christian Gruner, ${ }^{1}$ René Dominik Rötzer, ${ }^{1}$ Stefanie Fenske, ${ }^{1}$ Jana Hartmann, ${ }^{2}$ \\ Benedikt Zott, ${ }^{2}$ Anita Lüthi, ${ }^{4}$ Saskia Spahn, ${ }^{1}$ Markus Moser, ${ }^{3}$ Dirk Isbrandt, ${ }^{5}$ Andreas Ludwig, ${ }^{6}$ \\ Arthur Konnerth, ${ }^{2}$ Christian Wahl-Schott, ${ }^{1,7}$ and Martin Biel ${ }^{1}$ \\ 'Department of Pharmacy, Center for Drug Research, Center for Integrated Protein Science Munich, Ludwig-Maximilians- \\ Universität München, Munich, Germany. ${ }^{2}$ Institute of Neuroscience, Technical University of Munich, Munich, Germany; \\ and Munich Cluster for Systems Neurology (SyNergy) and Center for Integrated Protein Sciences (CIPSM), Munich, \\ Germany. ${ }^{3}$ Department for Molecular Medicine, Max-Planck-Institut für Biochemie, Martinsried, Germany. ${ }^{4}$ Department \\ of Fundamental Neurosciences, University of Lausanne, Lausanne, Switzerland. ${ }^{5} \mathrm{DZNE}$ Research Group, Experimental \\ Neurophysiology, Institute for Molecular and Behavioral Neuroscience, University of Cologne, Germany. IInstitut für \\ Experimentelle und Klinische Pharmakologie und Toxikologie, Friedrich-Alexander-Universität Erlangen-Nürnberg, \\ Erlangen, Germany. ${ }^{7}$ Institut für Neurophysiologie, Medizinische Hochschule Hannover, Hannover, Germany.
}

Hyperpolarization-activated cyclic nucleotide-gated (HCN) channels are dually gated channels that are operated by voltage and by neurotransmitters via the CAMP system. CAMP-dependent HCN regulation has been proposed to play a key role in regulating circuit behavior in the thalamus. By analyzing a knockin mouse model (HCN2EA), in which binding of CAMP to HCN2 was abolished by 2 amino acid exchanges (R591E, T592A), we found that CAMP gating of HCN2 is essential for regulating the transition between the burst and tonic modes of firing in thalamic dorsal-lateral geniculate (dLCN) and ventrobasal (VB) nuclei. HCN2EA mice display impaired visual learning, generalized seizures of thalamic origin, and altered NREM sleep properties. VB-specific deletion of HCN2, but not of HCN4, also induced these generalized seizures of the absence type, corroborating a key role of HCN2 in this particular nucleus for controlling consciousness. Together, our data define distinct pathological phenotypes resulting from the loss of cAMP-mediated gating of a neuronal HCN channel.

Conflict of interest: The authors have declared that no conflict of interest exists.

Copyright: (c) 2019 American Society for Clinical Investigation

Submitted: November 26, 2018 Accepted: April 2, 2019 Published: May 2, 2019.

Reference information: /CI Insight. 2019;4(9):e126418. https://doi. org/10.1172/ji.insight.126418.

\section{Introduction}

Hyperpolarization-activated cyclic nucleotide-gated $(\mathrm{HCN})$ channels are voltage-gated ion channels that conduct a current termed $I_{h}$, or $I_{f}$ (in heart) $(1,2)$. $I_{h}$ differs from almost all other currents characterized in neurons, as $I_{h}$ is activated upon membrane hyperpolarization (at potentials more negative than $-50 \mathrm{mV}$ ). The channels are open at rest and are important for the determination of the resting membrane potential (RMP) $(1,2)$. Moreover, HCN channels have been implicated in the regulation of other cellular and circuit functions, including synaptic transmission, dendritic integration, and neuronal rhythmicity (1). All 4 members of the HCN-channel family (HCN1-4) are expressed in the brain in only partly overlapping expression patterns, suggesting that each $\mathrm{HCN}$-channel subtype contributes to unique functions in a nonredundant fashion (3, 4). Knockout studies have principally confirmed this concept by elucidating specific roles for HCN1 (5-7) and HCN2 (8, 9). The function of $\mathrm{HCN} 3$ has been investigated in the heart (10) and brain (11) and the function of HCN4 has been studied in the heart (12). In contrast, the role of neuronal HCN4 channels remains to be elucidated.

A key feature of HCN channels is that their activation gating is facilitated by cAMP. Mechanistically, this modulation is conferred by cAMP binding to a domain in the C-terminus of HCN channels (the CNBD), which in turn induces a shift in the voltage dependence of activation towards more positive potentials (13-15). Consequently, at a given voltage, more current flows through an HCN channel if the cAMP concentration rises in a cell. Direct gating of HCN channels has been proposed to play a key role in heart rate regulation (16). With respect to the nervous system, an influence on the RMP and on the firing 
of action potentials (APs) by neurons due to the direct modulation of $\mathrm{I}_{\mathrm{h}}$ by cAMP was first demonstrated in the hippocampus (17). Since then, cAMP-mediated regulation of $I_{h}$ has been implicated in diverse processes such as in the transmission of inflammatory pain (18), regulation of spatial periodicity of grid cells in the medial entorhinal cortex (19), control of working memory (20), and coincidence detection of sound $(21,22)$. In particular, it has been proposed that cAMP-dependent regulation (CDR) of $\mathrm{I}_{h}$ is involved in the control of thalamic network activity (23-26). Thalamocortical (TC) neurons fire in 2 main modes: the burst firing mode, which is characterized by stereotypic short trains of high-frequency APs, and the tonic mode, in which the frequency of APs is correlated with the strength of an incoming depolarization through afferent excitatory inputs $(27,28)$. In the tonic mode, TC neurons can process sensory information (encoded by trains of APs) and forward it to the respective regions of the cortex. In contrast, the burst mode is linked to states in which thalamic information gating is reduced, for example, during deep sleep (27). It has been proposed that CDR of $I_{h}$ is a major mechanism whereby neurotransmitters regulate the transition from burst to tonic firing, and hence, the activity state of the thalamus $(26,27)$. However, the proposed model is mainly based on experiments using pharmacological tools. Experiments with global HCN2-knockout (HCN2-KO) mice (8) and rat models of epilepsy with an altered HCN-channel expression profile (WAG/ Rij) (29) support a key role of HCN channels in thalamic firing. However, direct proof that cAMP binding to the CNBD of $\mathrm{HCN}$ channels regulates firing is missing. Furthermore, TC neurons express 2 distinct $\mathrm{HCN}$ channel subtypes that are sensitive to cAMP, HCN2 and HCN4, and it is not known which of the two regulates TC activity $(3,8)$, or whether both channels cooperate in this regard. This question also extends to the role of thalamic HCN channel modulation in vigilance-state control, notably in sleep and in the transition to waking.

In the present study, we have directly addressed this question by analyzing an HCN2-knockin mouse model that expresses a mutant HCN2 channel (HCN2EA) with normal voltage-dependent gating, while CDR is completely abolished. We found that HCN2EA mice show impaired firing in 2 different thalamic nuclei, the dorsal lateral geniculate nucleus (dLGN) and the ventrobasal nucleus (VB). We also demonstrate that these defects directly translate into impaired visual learning, an altered spectral profile of non-rapid eye movement (NREM) sleep, and the generation of generalized seizures (spike and wave characteristics).

\section{Results}

Generation of HCN2EA mice and basic characterization of HCN2EA-mediated $I_{h}$. Using homologous recombination in embryonic stem cells we generated a knockin mouse model (HCN2EA) that expresses an HCN2 channel carrying 2 distinct amino acid substitutions in the CNBD (R591E and T592A) (Supplemental Figure 1; supplemental material available online with this article; https://doi.org/10.1172/jci.insight.126418DS1). The R591 and T592 residues are located in the loop between the P helix and the $\beta 7$ sheet of the CNBD and are highly conserved throughout the cyclic nucleotide-gated and HCN-channel family $(14,15)$. In high-resolution structures of the CNBD obtained for various cyclic nucleotide-modulated channels, both residues directly interact with the phosphate group of cAMP, indicating that these residues are required for high-affinity binding of cyclic nucleotides (Figure 1A). Mutation of R591 to glutamine or glutamate and/or mutation of T592 to alanine completely abolishes channel activation by cyclic nucleotides (30, 31). Indeed, when heterologously expressed in HEK293 cells, the HCN2EA channel revealed normal hyperpolarization-dependent gating, while modulation by cAMP (even at high millimolar concentrations) was completely lost $\left(\mathrm{HCN} 2: \mathrm{V}_{0.5}[\right.$ no cAMP $]=-100.60 \pm 0.60 \mathrm{mV}[n=9], \mathrm{V}_{0.5}[1 \mathrm{mM} \mathrm{cAMP}]=-89.50 \pm 0.79 \mathrm{mV}[n=$ 9]; HCN2EA: $\mathrm{V}_{0.5}$ [no cAMP] $=-101.10 \pm 0.75 \mathrm{mV}[n=7], \mathrm{V}_{0.5}[1 \mathrm{mM}$ cAMP $]=-98.96 \pm 2.07 \mathrm{mV}[n$ = 8]; data not shown). Heterozygous matings produced wild-type (WT), HCN2EA/WT, and HCN2EA/ EA mice at the expected Mendelian ratios. Here, the term HCN2EA is used to refer to mice that carry the 2 mutations on both Hcn2 alleles. HCN2EA mice showed no gross body abnormalities, nor did they differ from their WT littermates with regard to lifespan (Supplemental Figure 2A) or body weight (Supplemental Figure 2B). Unlike HCN2-KO mice, the HCN2EA mice had neither whole-body tremor nor ataxia (Supplemental Figure 2, C and D) $(8,9)$. The overall brain morphology of HCN2EA mice appeared normal (Figure 1B). We examined the expression of HCN channels in the thalamus using immunohistochemistry and Western blot analysis. Western blots and quantitative reverse transcription PCR (qRT-PCR) confirmed that the dLGN expresses only HCN2, whereas the VB region contains both HCN2 and HCN4 (Figure 1C and Supplemental Figure 3D). We next compared expression levels of WT and HCN2EA channels. Western blot analysis of membrane fractions from whole brain revealed no difference in protein amounts between the 
2 genotypes (Figure 1, D and E). Importantly, we also found evidence for the formation of heterotetrameric $\mathrm{HCN} 2 / \mathrm{HCN} 4$ and HCN2EA/HCN4 channels in whole-brain membrane fractions (Supplemental Figure $3 \mathrm{~A}$ ), indicating that the principal channel architecture of HCN2 is not disturbed by the EA mutation. In agreement with this finding, HCN2EA channels interacted like WT channels with the auxiliary HCN-channel subunit TRIP8b $(32,33)$ (Supplemental Figure 3B). Staining of horizontal brain slices from WT mice showed broad expression of HCN2 throughout the thalamus (Figure 2A). In the VB region, a strong signal for HCN4 was detected. However, HCN4 was not detected in the dLGN (Figure 2B). The expression of $\mathrm{HCN} 1$ in the thalamus (VB and dLGN) was below the detection level (Supplemental Figure 3C). HCN3 is expressed in the intergeniculate leaflet, while there is no expression in the VB (11). Further characterization of HCN2 channel expression in the VB region from staining in neurons revealed that expression levels of HCN2 were higher in somata as compared with dendrites (Figure 2, C and D). Importantly, however, the distribution of HCN2 was not different between WT and HCN2EA mice (Figure 2, C and D). In agreement with this finding, analysis of the expression of HCN2 in primary neurons from WT and HCN2EA mice revealed no difference in the expression level along dendrites and in somata (Supplemental Figure 4). Expression of HCN4 was slightly enriched in dendrites and was also not different between WT and HCN2EA animals (Figure 2, E and F).

We next characterized $I_{h}$ in TC neurons from thalamic slice preparations. Figure $3 \mathrm{~A}$ shows representative traces of whole-cell currents from VB TC neurons of WT and HCN2EA. The shape of the current traces was identical; however, the opening kinetics of the HCN2EA current were somewhat slower compared with those of WT ( $\tau$ at $-140 \mathrm{mV}$ : WT, $166.46 \pm 11.29 \mathrm{~ms}[n=7]$; HCN2EA, $252.66 \pm 9.09 \mathrm{~ms}[n=$ 8]; data not shown). The current density in the dLGN was similar for HCN2EA compared to WT (Figure 3B; current density at $-140 \mathrm{mV}$ : WT, $6.45 \pm 0.55 \mathrm{pA} / \mathrm{pF}$; HCN2EA, $6.65 \pm 0.34 \mathrm{pA} / \mathrm{pF}$ ). In the VB region current densities were generally higher than in the dLGN but were in the same range for WT and HCN2EA (current density at $-140 \mathrm{mV}$ : WT, $15.02 \pm 1.96 \mathrm{pA} / \mathrm{pF}$; HCN2EA, $12.23 \pm 0.87 \mathrm{pA} / \mathrm{pF}$ ). These experiments suggest that cAMP does not affect current density and maximal conductance of $\mathrm{I}_{\mathrm{h}}$. In agreement with these findings, maximal current density and maximal conductance were similar in TC neurons recorded in acute slice preparations (Supplemental Figure 5A). This conclusion was further confirmed by planar patch-clamp experiments of HEK293 cells stably expressing WT or HCN2EA channels (Supplemental Figure 5B). These experiments were carried out in a special configuration that allows for exchange of the intracellular solution with the cytosolic solution. In these experiments, we applied intracellular control solution and subsequently exchanged it with a solution containing cAMP through perfusion (Supplemental Figure 5B, inset). Together, the experiments in both TC neurons and HEK293 cells expressing HCN2 channels (Supplemental Figure 5, $\mathrm{C}$ and $\mathrm{D}$ ) indicate that cAMP does not increase channel conduction and current densities. We next determined the voltage dependence of activation of $I_{h}$ in the absence of cAMP and after perfusion with $1 \mu \mathrm{M}$ cAMP, which is close to the reported apparent cAMP affinities of $I_{h}$ in neurons (1). Figure 3C shows activation curves for TC neurons of dLGN from HCN2EA and WT. Unlike WT, which displayed a significant shift in the $\mathrm{V}_{0.5}\left(\Delta \mathrm{V}=+5.05 \mathrm{mV}: \mathrm{V}_{0.5}\right.$ no cAMP, $\left.-94.57 \pm 0.77 \mathrm{mV} ;+\mathrm{cAMP},-89.51 \pm 1.31 \mathrm{mV}\right)$, the voltage-dependent activation of $\mathrm{HCN} 2 \mathrm{EA}$ was not affected by cAMP $\left(\Delta \mathrm{V}=+1.10 \mathrm{mV}\right.$ : $\mathrm{V}_{0.5}$ no cAMP, -99.97 $\pm 0.80 \mathrm{mV} ;+\mathrm{cAMP},-98.87 \pm 0.43 \mathrm{mV})$. In the VB region of HCN2EA, voltage-dependent activation of $\mathrm{I}_{\mathrm{h}}$ was still modulated by cAMP, although to a significantly lesser extent than the WT. HCN2EA $(\Delta \mathrm{V}=+3.59$ $\mathrm{mV}: \mathrm{V}_{0.5}$ no cAMP, $\left.-104.11 \pm 0.77 \mathrm{mV} ;+\mathrm{cAMP},-100.52 \pm 0.63 \mathrm{mV}\right)$, WT $\left(\Delta \mathrm{V}=+5.56 \mathrm{mV}: \mathrm{V}_{0.5}\right.$ no cAMP, $-97.92 \pm 0.55 \mathrm{mV} ;+\mathrm{cAMP},-92.36 \pm 0.31 \mathrm{mV})$. At a saturating cAMP concentration $(100 \mu \mathrm{M})$ the voltage shift was $+18.3 \mathrm{mV}$ for $\mathrm{WT}$ and $+6.6 \mathrm{mV}$ for HCN2EA. These data are consistent with the expression data showing that in the dLGN, HCN2 is the only channel expressed whereas VB neurons contain HCN2 plus HCN4, which could contribute to the residual cAMP sensitivity of $I_{h}$ in HCN2EA mice.

Impaired firing in the $d L G N$ and reduced visual learning in HCN2EA mice. We examined the intrinsic firing properties of WT TC neurons from whole-cell current-clamp measurements. In the absence of cAMP, TC neurons responded to injections of positive current at the RMP with the generation of high-frequency burst discharges (Figure 4, A and B). When measurements were performed in the presence of $1 \mu \mathrm{M}$ cAMP in the pipette and a slight depolarization was induced, cells fired a tonic series of APs. In contrast, within the range from 50-200 pA of injected current, TC neurons from HCN2EA mice fired in the burst mode independently of the absence or presence of CAMP (Figure 4, A and B). These neurons produced tonic series of APs only in response to a strong depolarizing current injection (400 pA) (Figure 4B) or if the RMP was elevated to WT conditions (Supplemental Figure 6). As mentioned, we observed that the loss of cAMP regulation in 
A

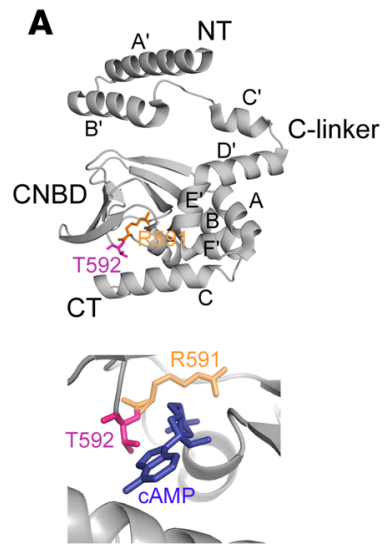

B
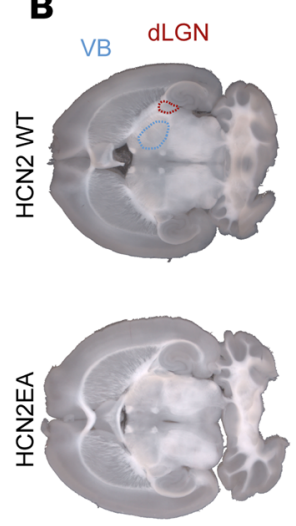

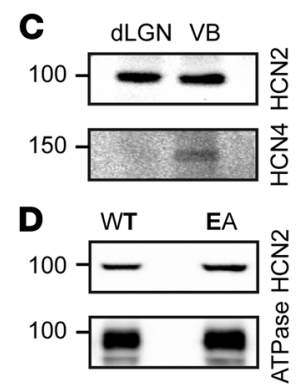

E

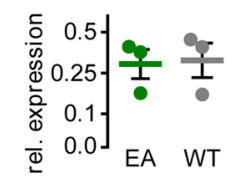

Figure 1. Impaired modulation of $\mathrm{I}_{\mathrm{h}}$ by CAMP in thalamocortical neurons expressing HCN2EA. (A) Structural model of the CNBD of HCN channels. The 2 key residues R591 (yellow) and T592 (pink) that are crucial for binding of cAMP are highlighted. (B) Horizontal brain slices of WT and HCN2EA mice. The position of the dLGN (red) and the VB (blue) is indicated. (C) Detection of HCN2 and HCN4 in Western blot analysis of punched dLGN and VB regions. Images are representatives of $n=3 /$ group. (D) Western blot analysis of membrane preparations of HCN2EA and WT mice probed for HCN2 and a loading control ( $\mathrm{Na}^{+} / \mathrm{K}^{+}$-ATPase). Images are representatives of $n=3 /$ group. (E) Quantification of HCN2 expression level in relation to the $\mathrm{Na}^{+} / \mathrm{K}^{+}-\mathrm{ATPase}(n=3)$.

HCN2 had an effect on the RMP. The RMP of HCN2EA TC neurons differed in 2 aspects from that of WT (Figure 4C). First, it was hyperpolarized in comparison with WT (HCN2EA, $-84.99 \pm 1.08 \mathrm{mV}$; WT, $-81.46 \pm 0.72 \mathrm{mV}$ ) and second, it was not depolarized by cAMP (HCN2EA, $-84.47 \pm 0.76 \mathrm{mV} ; \mathrm{WT},-76.69$ $\pm 0.71 \mathrm{mV}$ ). This finding supports a key role for cAMP-dependent regulation of HCN2 in the determination of the RMP in dLGN TC neurons. The consequences of the cAMP regulation could also be measured by the voltage sag, which is a characteristic feature of $I_{h}$ (Figure 4D). WT neurons revealed a prominent, slow, time-dependent rectification during hyperpolarization that is caused by the activation of $\mathrm{I}_{\mathrm{h}}$. In HCN2EA mice the voltage sag was less pronounced (i.e., the ratio between the peak and steady-state voltages was closer to 1) and unlike in WT, was independent of cAMP. Taken together, these findings support a key role for cAMP modulation of HCN2 in regulating the basic firing properties of TC neurons in the dLGN. Because the dLGN is the main thalamic relay center of the primary visual pathway from retina to cortex $(34,35)$ (Figure 5A), we examined visual learning in WT and HCN2EA mice using a visual discrimination test that is sensitive to thalamic information processing via the geniculate pathway (Figure 5B). This test revealed that the visual learning capability in HCN2EA mice is significantly reduced during the first 2 test days. Only at later time points did both genotypes show comparable results in their performance (Figure 5B). In principle, reduced visual learning could simply reflect a defect in retinal function. To test this hypothesis, we performed a virtual-cliff test (Figure 5C), which allows the visual function to be evaluated. In this test, HCN2EA and WT mice performed equally well, whereas triple-KO mice, which are deficient in cyclic nucleotide-gated

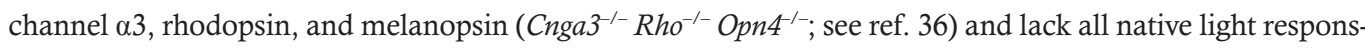
es driven by photoreceptors or photosensitive ganglion cells, failed to pass the test (Figure 5C). Finally, to demonstrate that the learning defect observed in HCN2EA mice reflects a specific failure in thalamic function, we used a Morris water-maze paradigm to test hippocampus-dependent spatial learning. In this test, HCN2EA and WT mice performed equally well (Figure 5D).

Role of $C A M P$ modulation of HCN2 in the VB region. We also extensively studied $\mathrm{I}_{\mathrm{h}}$ in the VB complex, which is the main relay nucleus for somatosensory stimuli. Qualitatively, loss of cAMP modulation of HCN2 had the same impact on the firing properties of TC neurons in the VB as in the dLGN (Figure 6, $\mathrm{A}$ and $\mathrm{B}$ ). Under basal (cAMP-free) conditions, WT neurons fired in the burst mode and switched to tonic firing upon stronger stimuli (200 pA). In contrast, in the presence of $1 \mu \mathrm{M}$ cAMP, TC neurons did not reveal burst activity, but consistently produced tonic series of APs. HCN2EA neurons only produced bursts of APs and showed tonic firing at very strong stimuli (400 pA) (Figure 6, A and B). The RMP of TC neurons of HCN2EA was hyperpolarized compared with WT (HCN2EA, $-82.85 \pm 0.67 \mathrm{mV}$; WT, $-77.19 \pm 0.65 \mathrm{mV}$ ) (Figure 5C). In agreement with the presence of a residual cAMP-dependent $\mathrm{I}_{\mathrm{h}}$ current (Figure 3C), the RMP of HCN2EA TC neurons became more depolarized in the presence of $1 \mu \mathrm{M}$ cAMP $(-79.71 \pm 0.77 \mathrm{mV})$ 


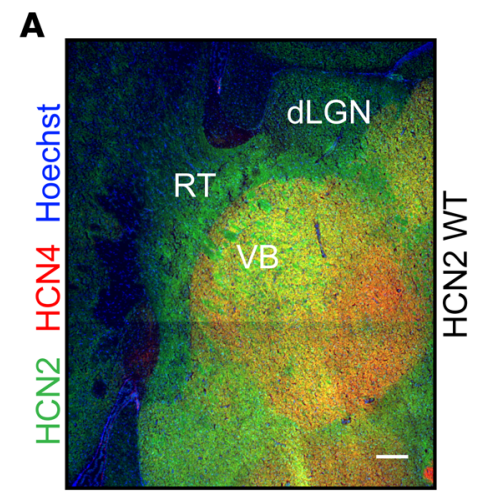

\section{B}
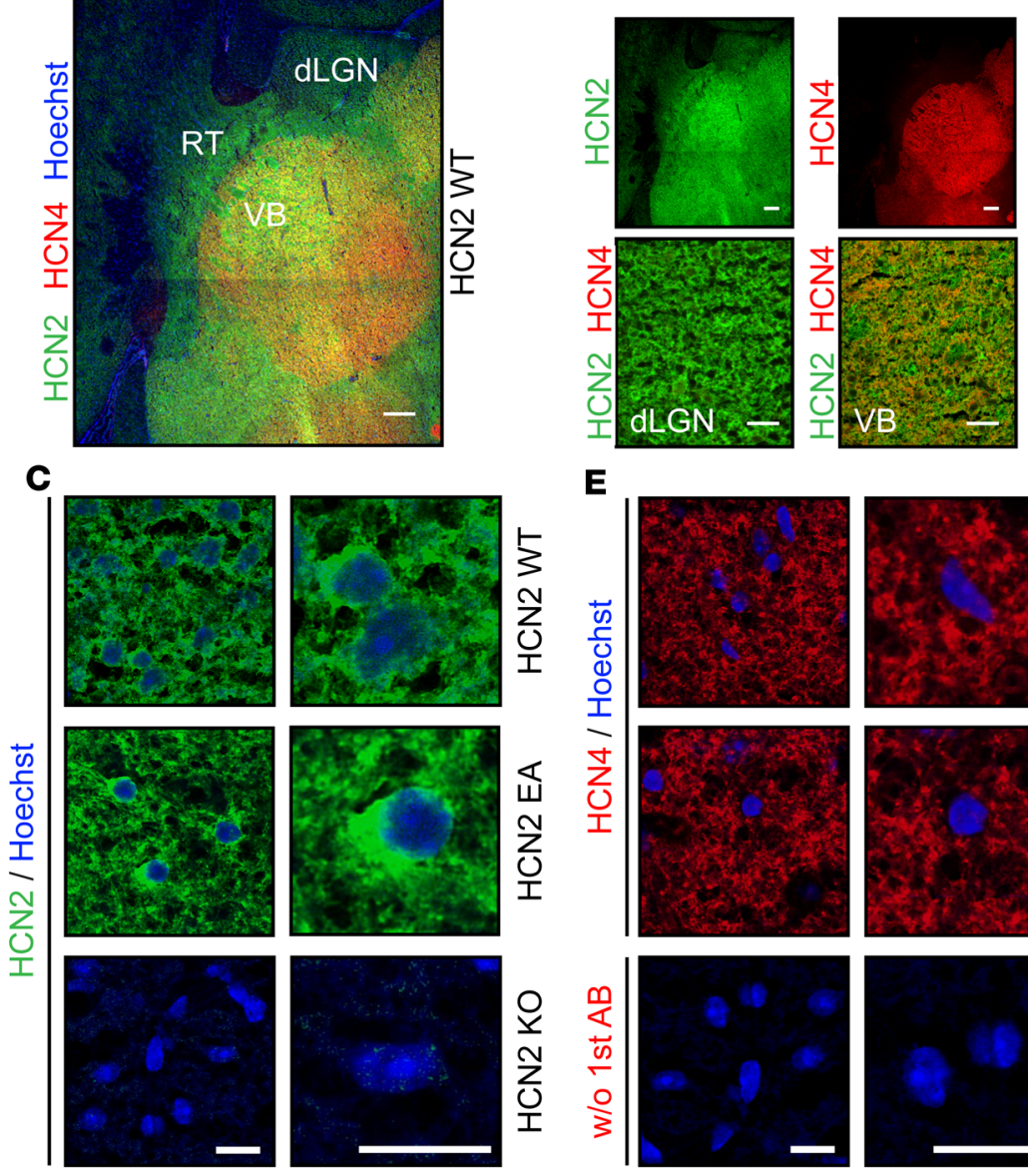

$\mathbf{E}$
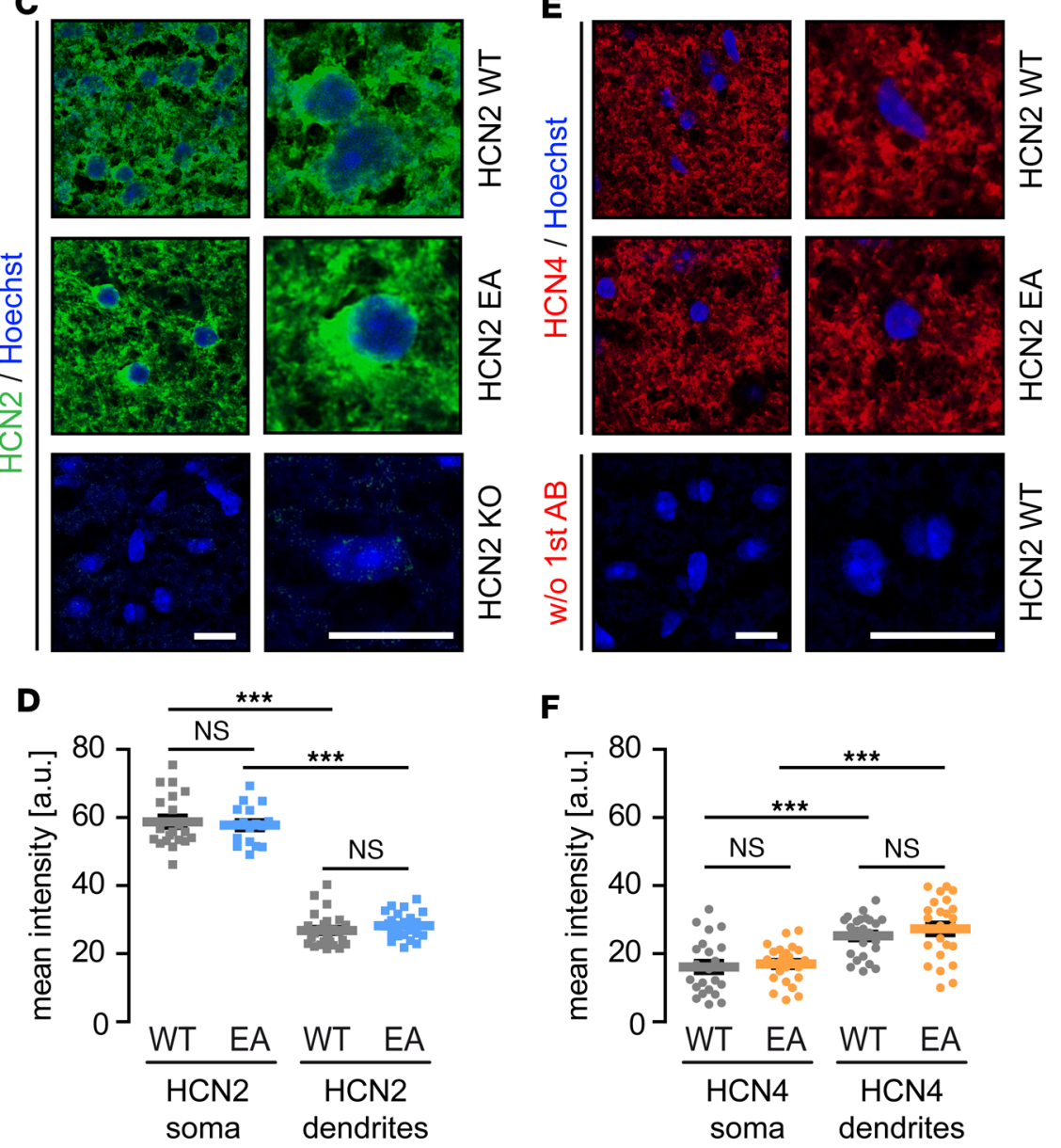

Figure 2. HCN2 and HCN4 staining in the thalamus. (A) Distribution of HCN2 and HCN4 in the VB region of mouse thalamus. Scale bar: $200 \mu \mathrm{m}$. (B) Overlay of anti-HCN2 (green), anti-HCN4 (red), and Hoechst (blue). Scale bars: $200 \mu \mathrm{m}$. Upper: Single channels for HCN2 (green) and HCN4 (red) staining. Lower: Magnification (scale bars: 25 $\mu \mathrm{m}$ ) of the dLGN (left) and VB (middle). (C) Magnified HCN2 stainings of WT (upper), HCN2EA litters (middle), and HCN2-KO mice (lower panel) in the VB region. Scale bars: $20 \mu \mathrm{m}$. (D) Analysis of the mean intensity of the HCN2 fluorescence in WT and HCN2EA soma and dendrites (WT: gray squares, soma [ $n=20]$, dendrites [ $n=26]$; EA: blue squares, soma [ $n=17]$, dendrites $[n=26]$ ). (E) Magnified HCN4 stainings of WT (upper) and HCN2EA litters (middle). Staining in the absence of primary antibody is shown in the bottom panels. Scale bars: $20 \mu \mathrm{m}$. (F) Analysis of the mean intensity of the HCN4 fluorescence in WT and HCN2EA soma and dendrites (WT: gray circles, soma [ $n$ $=23]$, dendrites $[n=25]$; EA: orange circles, soma $[n=25]$, dendrites $[n=25])^{* * *} P<0.001$ by 1 -way ANOVA with Bonferroni's post hoc test. NS, not significant. 

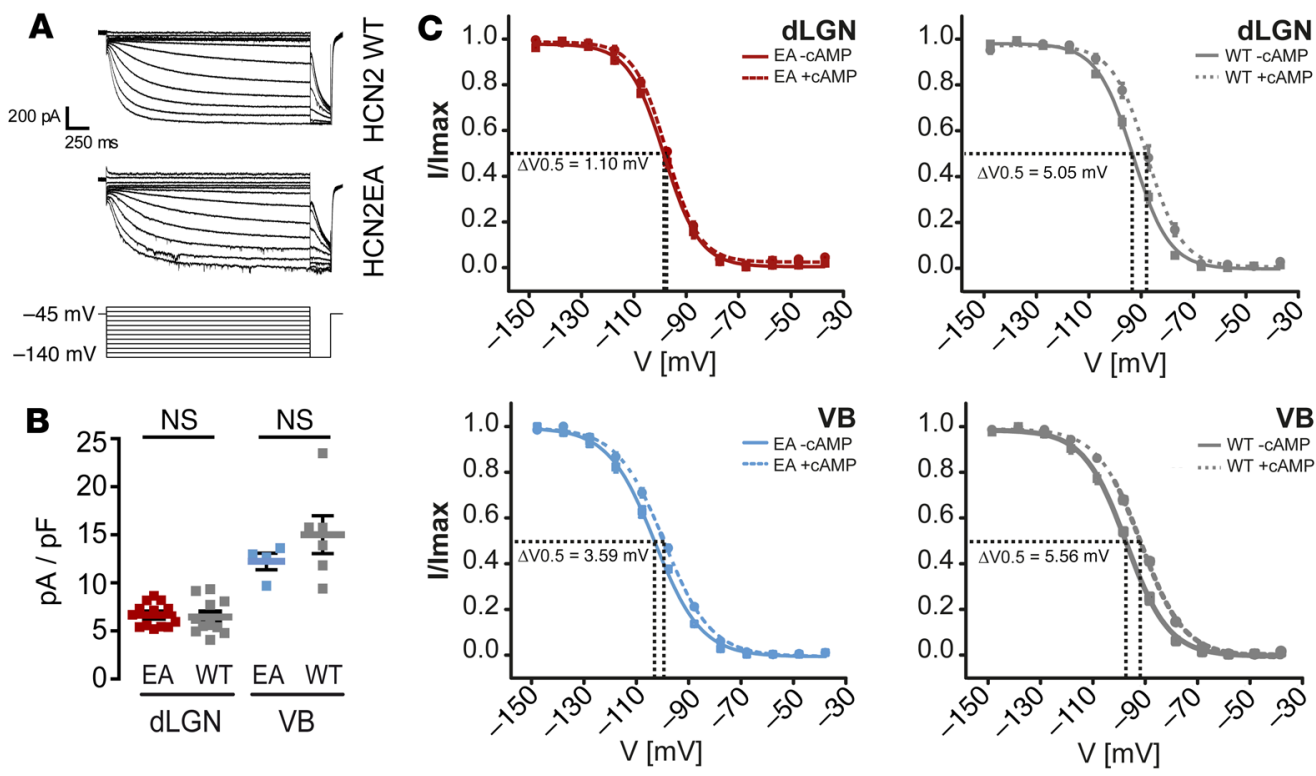

Figure 3. Impaired modulation of $\mathrm{I}_{h}$ by cAMP in thalamocortical neurons expressing HCN2EA. (A) Representative $\mathrm{I}_{h}$ traces from thalamocortical WT (upper) and HCN2EA neurons (lower) in the VB. Below, voltage protocol used to evoke current traces. (B) $I_{h}$ density of thalamocortical neurons in the dLGN (HCN2EA, red squares $[n=13]$; WT, gray squares $[n=$ 11]; $P=0.3524$ by Mann-Whitney test) and the VB (HCN2EA, blue squares [ $n=4]$; WT, gray squares $[n=6] ; P=0.6021$ by Mann-Whitney test). NS, not significant. (C) Normalized current-voltage relationships of $I_{\mathrm{h}}$ in thalamocortical neurons of WT and HCN2EA mice in the absence (-CAMP) and presence of $1 \mu \mathrm{M}$ cAMP (Boltzmann fit). Upper left: dLGN, HCN2EA: solid red line, -cAMP $(n=6)$; dashed red line, +CAMP $(n=4)$. Upper right: dLGN, WT: solid gray line, -cAMP $(n=6)$; dashed gray line, + CAMP $(n=5)$. Lower left: VB, HCN2EA: solid blue line, -cAMP $(n=4)$; dashed blue line, + CAMP $(n=5)$. Lower right: VB, WT: solid gray line, -cAMP $(n=6)$; dashed gray line, + CAMP $(n=8)$. The cAMP-dependent shift of the midpoint potentials $\left(V_{0.5}\right)$ of activation is indicated as dashed black lines.

(Figure 6C). Similarly, HCN2EA neurons revealed a larger voltage-sag ratio (i.e., weaker contribution of $\mathrm{I}_{\mathrm{h}}$ ) than in WT neurons; however, the voltage sag was slightly increased by cAMP (Figure 6D).

To examine the effect of altered firing behavior on network activity, we used a 2-photon $\mathrm{Ca}^{2+}$ imaging approach as previously described for the analysis of HCN2-KO mice (8). Spontaneous discharge patterns of TC cell ensembles in the semipreserved thalamic network were investigated in horizontal slices of somatosensory thalamus in which spontaneous rhythmicity is high due to cell-intrinsic and intrathalamic mechanisms (cell-intrinsic bursting of TC cells) (37-39). Given this connectivity, intrathalamic reverberating low-frequency oscillations can be induced. As a measure of electrical activity, $\mathrm{Ca}^{2+}$ fluctuations of ensembles of TC neurons were monitored. Upon facilitation of neuronal activity by 4 -aminopyridine (4-AP), 74 out of 153 WT cells showed spontaneous calcium transients (Figure 6E). Oscillatory behavior was not detected in any of these cells (Figure 6F). In preparations from HCN2EA mice, 70 out of 170 cells were active in the presence of 4-AP. However, more than $35 \%$ of these cells showed oscillatory activity (Figure 6, E and F), as evident by the repeated synchronous increases in fluorescence at approximately 0.5 $\mathrm{Hz}$, a frequency that is typical for the clock-like delta rhythm of TC cells in the acute slice $(26,38)$.

Loss of cAMP modulation in HCN2 is sufficient to produce generalized seizures. In order to determine the consequences of altered thalamic network activity we performed telemetric EEG recordings in freely moving mice (Figure 7, A and B). HCN2EA mice displayed frequent spike-and-wave discharges (SWDs) and behavioral arrest, the hallmark of generalized seizures of the absence-epilepsy type (Figure 7A and Supplemental Videos 1-3). The SWDs concurred with episodes of low muscular tone as reflected in the EMG (traces below the EEG). The SWDs had a frequency of $3-5 \mathrm{~Hz}$ and comprised $0.30 \% \pm 0.11 \%$ of the recording time. The mean duration of an SWD was $4.57 \pm 0.50$ seconds and the time between 2 SWDs was 2,175 \pm 734.6 seconds (Figure 7B). Global HCN2-KO mice displayed SWDs with comparable frequency and duration as seen in HCN2EA mice (8). In contrast, SWDs were not detected in WT littermates.

Next, we examined the time spent in the different vigilance states (wake, REM sleep, NREM sleep). In animals entrained to a 12-hour light/12-hour dark cycle, both WT and HCN2EA mice showed a sleep-wake pattern characteristic of nocturnal animals with their main resting period during the light phase, in which they 


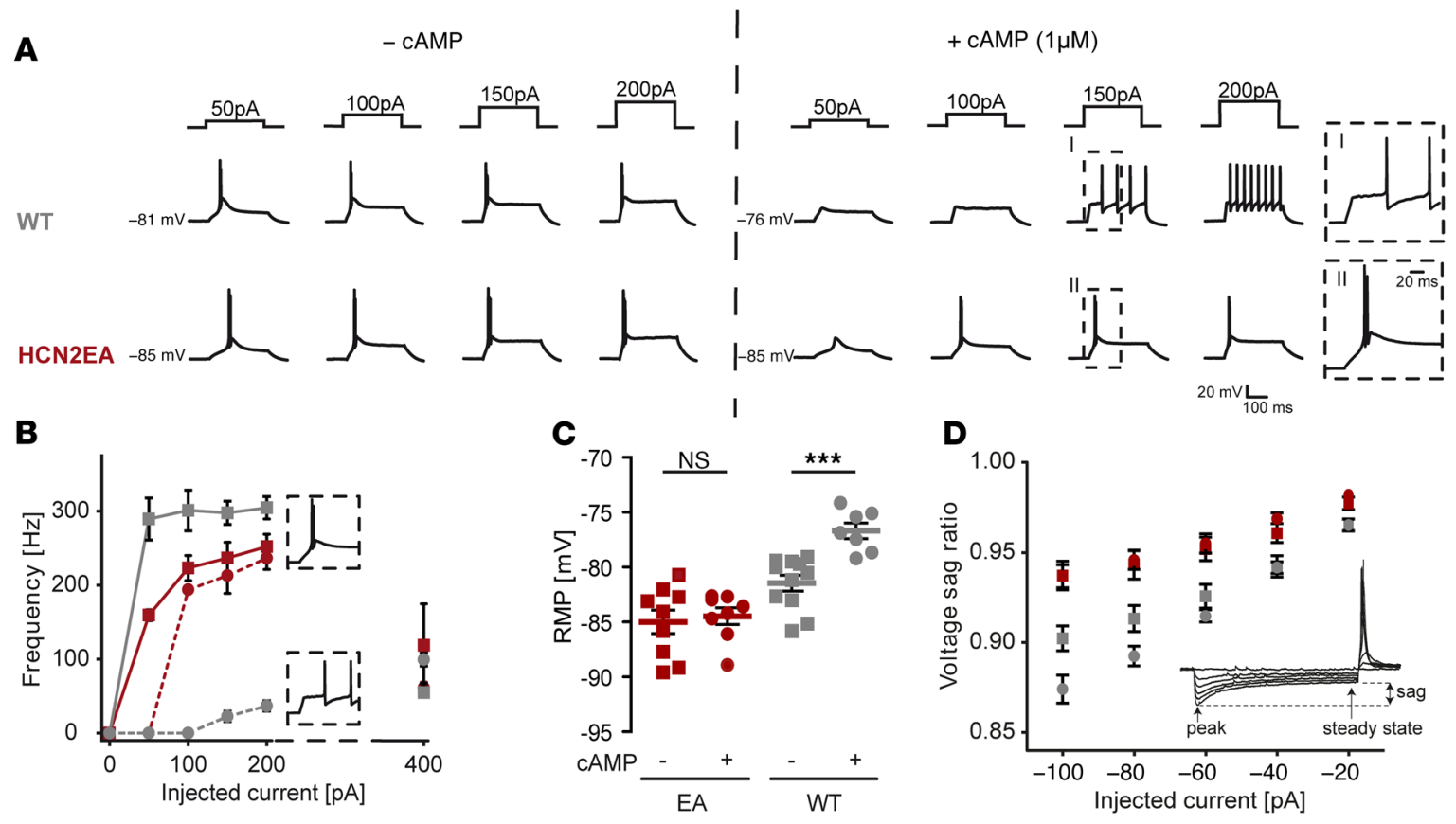

Figure 4. Lack of CAMP modulation in HCN2 alters firing properties of thalamocortical neurons in the dLGN. (A) Firing properties of WT and HCN2EA dLGN neurons in the absence (-cAMP, left) or presence of $1 \mu \mathrm{M}$ cAMP (right). Current injections $(300 \mathrm{~ms})$ are indicated above the traces. Insets: Enlarged traces elicited at $150 \mathrm{pA} /+\mathrm{CAMP}$ for WT (I) and HCN2EA (II). Traces are representatives of measurements shown in B. (B) Firing frequencies of TC neurons at different injected currents. HCN2EA (solid red line, -cAMP [ $n=5]$; dashed red line, + CAMP [ $n=3]$ ); WT (solid gray line, $-c A M P[n=4]$; dashed gray line, + CAMP $[n=3]$ ). Insets: Representative examples of burst and tonic firing at 200 pA. When injecting 400 pA, neurons fired in tonic mode independently of genotype and cAMP concentration. (C) Resting membrane potential (RMP) of TC neurons in the absence and presence of $1 \mu \mathrm{M}$ cAMP. HCN2EA (red squares, -cAMP $[n=9]$; red circles, + CAMP $[n=8]$ ). WT (gray squares, - cAMP $[n=11]$; gray circles, + cAMP $[n=7])$. NS, $P=0.7052\left(\right.$ EA), ${ }^{* * *} P=0.0004($ WT) by unpaired $t$ test. (D) Voltage-sag ratios (peak vs. steady state) at different negative current injections in the absence and presence of $1 \mu M$ cAMP. HCN2EA (red squares, - cAMP $[n=9]$; red circles, + cAMP $[n=8]$ ); WT (gray squares, - cAMP $[n=11]$; gray circles, + cAMP $[n=7]$ ). Inset: Example measurement of the voltage sag and calculation of the sag ratio.

spend approximately $70 \%$ of their time asleep (NREM and REM sleep). Both genotypes also did not differ with respect to the total time spent in the different vigilance states during the light and the dark phases (Figure 7C). However, mean normalized EEG spectral profiles from 0 to $25 \mathrm{~Hz}$ differed specifically for NREM but not for REM sleep (data not shown) in frequency bands known to arise within thalamocortical loops (Figure 7D). First, the sigma power band $(9-16 \mathrm{~Hz})$ that contains sleep spindles (40) was attenuated in the HCN2EA mice (Figure 7E; WT, $0.78 \pm 0.17$ vs. HCN2EA, $0.61 \pm 0.05$ ). The observed attenuation of sigma power was equally distributed across the power band. Conversely, the power in the delta band was not equally distributed. It was markedly augmented in the low-frequency slow delta $\left(\delta_{1}: 1.75 \mathrm{~Hz}\right)$, while the fast delta band $\left(\delta_{2}: 2.5-4\right.$ $\mathrm{Hz}$ ) was unchanged. Because sleep spindles and delta rhythms depend on rhythmic activity in TC neurons, this result strongly suggests that increased cell-autonomous burst firing of TC neurons in HCN2EA mice boosted the occurrence of low-frequency thalamocortical rhythms in the EEG, yet compromised the capability of these cells to engage in reciprocal interactions with thalamic reticular nucleus (nRT) cells to generate sleep spindles.

We wondered whether impaired VB complex firing caused the absence-seizure phenotype observed in HCN2EA. To verify this hypothesis, we locally deleted the HCN2 channel in the VB by stereotactically injecting an AAV2/8 vector expressing Cre recombinase under the control of neuron-specific human synapsin promoter into the VB of $H C N 2^{f / f l}$ mice. Using coexpressed EGFP as a reporter, we verified that only cells in the VB, but not cells in the thalamic RT or ventromedial regions were transfected and that these cells do not have HCN2 channel expression (Figure 8, A and B). EEG measurements performed 2 weeks after injection consistently revealed the presence of SWDs that were qualitatively similar to those found in global HCN2-KO or HCN2EA mice (Figure 8C). In mice injected with an adeno-associated virus (AAV) that only expressed EGFP, SWDs were absent, indicating that the effect was caused by the deletion of HCN2 
A

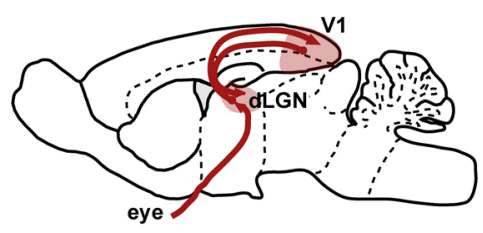

C

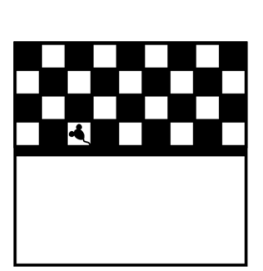

B

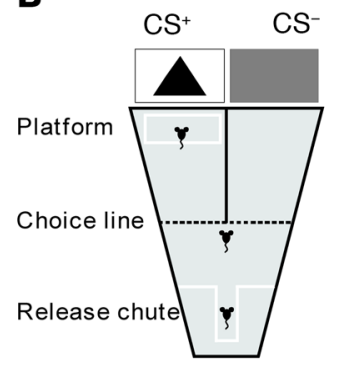

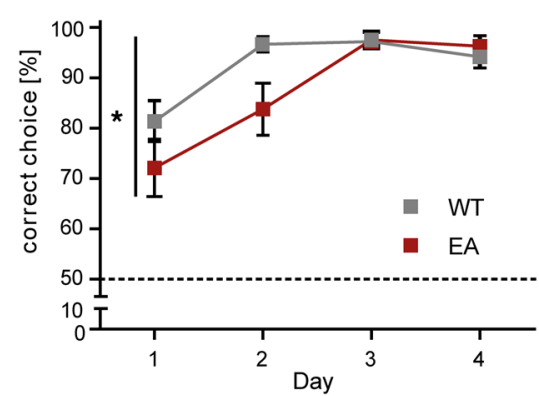

D wT
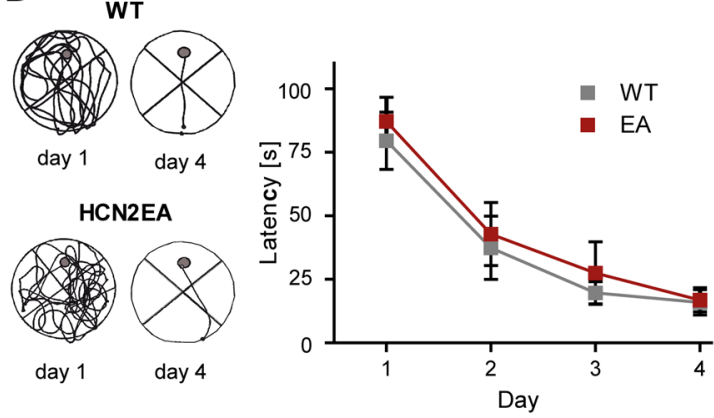

Figure 5. Lack of cAMP modulation in HCN2 impairs visual learning. (A) Scheme of the geniculate pathway. The dLGN receives input directly from the retina and projects towards the primary visual cortex (V1). (B) Left: Design of the visual discrimination task. CS', conditioned stimulus; CS $^{-}$, nonconditioned stimulus. Right: Learning curve of HCN2EA (red) and WT (gray) animals $(n=8)$. ${ }^{*} P<0.05$ by 1 -way ANOVA with Bonferroni's post hoc test. Dashed line

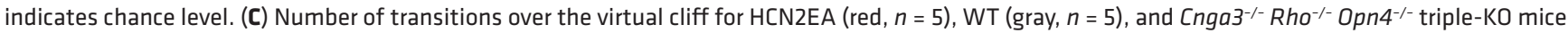
(black, $n=4$ ). ${ }^{* *} P<0.01$ by 1-way ANOVA with Bonferroni's post hoc test. NS, not significant. (D) Swimming traces of WT (upper left) and HCN2EA mice (lower left) on day 1 and day 4 in a Morris water maze. Right: Latency of WT (gray, $n=9$ ) and HCN2EA mice (red, $n=9$ ) to find a hidden platform.

in the VB (Figure 8D). Finally, we sought to determine whether VB-specific deletion of HCN4 could also exert effects on the EEG, given that HCN4 is coexpressed in the VB together with HCN2 and likely underlies the residual cAMP-sensitive $\mathrm{I}_{\mathrm{h}}$ component observed in HCN2EA. However, in the EEG of HCN4//f mice injected with the same viral vector used for HCN2 deletion, as well as in $H C N 4^{l / f}$ mice injected with an EGFP-expressing control vector, we did not observe SWDs (Figure 8E).

\section{Discussion}

It is known that cAMP is a crucial factor regulating $\mathrm{I}_{\mathrm{h}} / \mathrm{HCN}$ channel function in the heart and brain. However, so far it has not been possible to directly identify functions attributable to CDR of HCN channels in vivo without interfering with voltage-dependent functions. Most studies addressing CDR of $\mathrm{HCN}$ channels have been based on pharmacological blockers of $\mathrm{I}_{\mathrm{h}}$, or on the use of experimental approaches that involve up- or downregulation of cAMP-signaling pathways upstream of the channels. One study prevented CDR of HCN channels using a synthetic peptide based on TRIP8b (41). However, this peptide can only be used for in vitro experiments and is also not specific for a given $\mathrm{HCN}$ channel isoform. In the present study, we aimed to directly address this problem by genetically abolishing cAMP binding in the HCN2 channel without altering any other principal channel features. This strategy allowed us to define the specific contribution of cAMP modulation for a distinct member of the HCN-channel family (HCN2). Nevertheless, it might be possible that altered cAMP dynamics could interfere with our phenotype by pathways that do not involve direct cAMP-dependent gating of $\mathrm{HCN} 2$.

How do changes in CDR of HCN2 lead to changes in neuronal function, neuronal networks, and behavior? To address this question we focused on the thalamocortical circuit. In the current study, we show direct evidence that loss of CDR has profound consequences for the switch between tonic to burst-firing in thalamic neurons. We found that HCN2EA TC neurons of both dLGN and VB nuclei display a slightly hyperpolarized RMP compared with WT, indicating that basal cAMP levels in these neurons normally lead to a sustained partial preactivation of HCN2. The difference between WT and HCN2EA RMP was less pronounced in the VB, likely because VB neurons (in contrast to the dLGN) also express the highly 

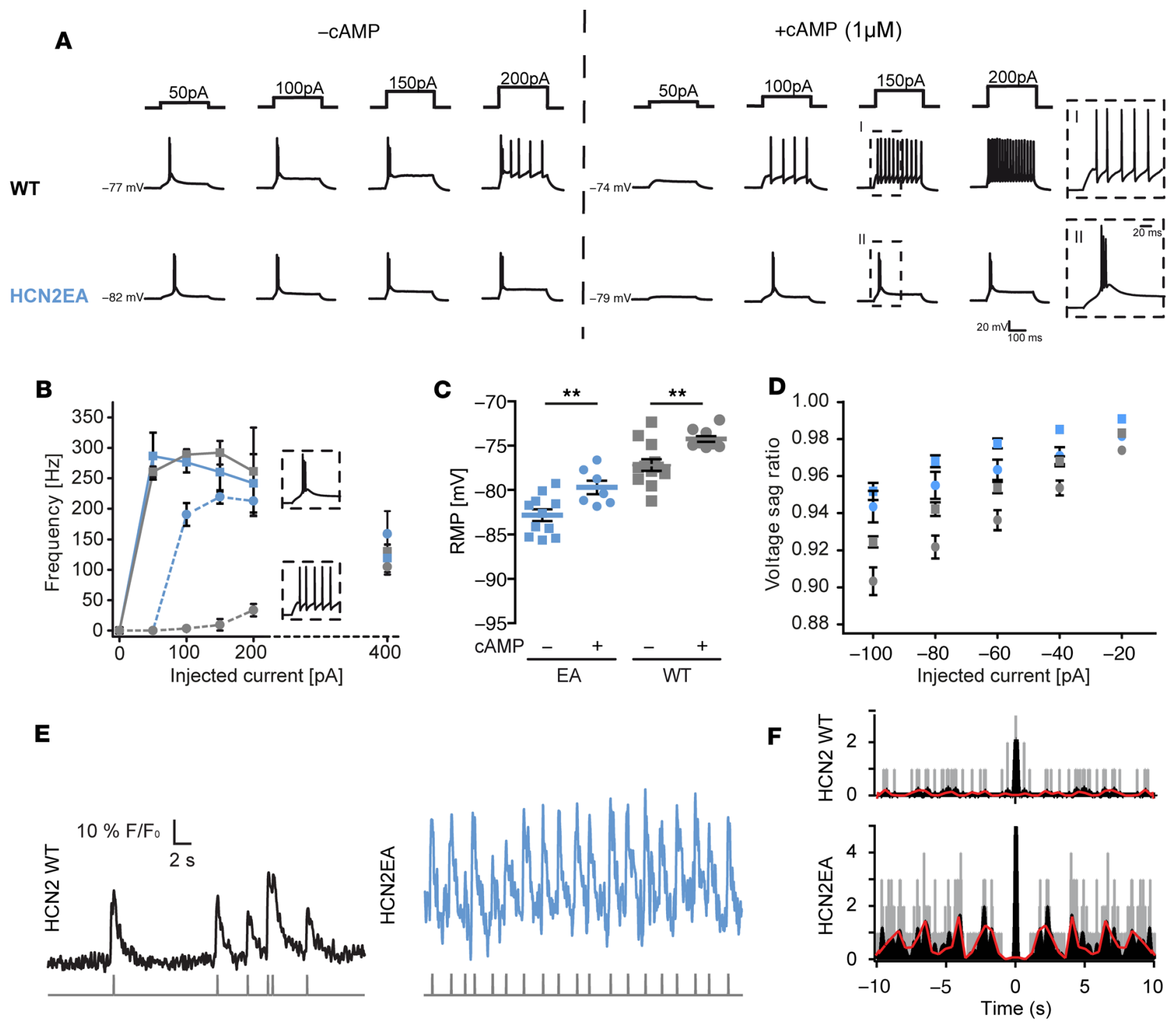

Figure 6. Analysis of neuronal network activity in the thalamic VB region. (A) Firing properties of WT and HCN2EA TC neurons from the VB region. Shown are action potentials elicited by 300-ms depolarizing current pulses at resting membrane potential of WT and HCN2EA TC neurons under control conditions (-CAMP) and in the presence of $1 \mu \mathrm{M}$ cAMP. Unlike WT neurons, HCN2EA neurons fail to switch from burst to tonic firing in the presence of cAMP within the current range from 50-200 pA. The insets show representative examples of tonic (I) and burst (II) firing activity induced by injecting $150 \mathrm{pA}$ in a WT and HCN2EA neuron, respectively. Traces are representatives of measurements shown in B. (B) Frequency of action potential generation as a function of the injected current. HCN2EA: solid blue line, $-\operatorname{cAMP}(n=6)$; dashed blue line, + CAMP $(n=6)$. WT: solid gray line, - cAMP $(n=4)$; dashed gray line, + cAMP $(n=5)$. (C) Resting membrane potential (RMP) of TC neurons from the VB of HCN2EA (blue squares, - CAMP $[n=11]$; blue circles, + CAMP [ $n=7]$ ) and WT (gray squares, -CAMP $[n=13]$; gray circles, + CAMP $[n=10])$.** $P<0.01(P=0.0083$ [EA], $P=0.0013$ [WT]) by Mann-Whitney test. (D) Voltage-sag ratios of TCs in response to injected current. HCN2EA (blue squares, -cAMP $[n=11]$; blue circles, + cAMP $[n=7]$ ); WT (gray squares, - cAMP [ $n=13]$; gray circles, + CAMP $[n=10]$ ). (E) Original traces of spontaneous activity of individual thalamic neurons visualized using 2-photon calcium imaging. The raster plots under the traces (gray) indicate the detected peaks. All active cells from WT mice show irregular transients (left, $n=74$ ), whereas more than one-third of the active cells from HCN2EA mice show oscillatory transients (right, $n=25$ ). (F) Autocorrelograms of the traces shown in $\mathbf{E}$. Gray, original autocorrelation function (ACF); black, smoothed ACF; red, cubic spline interpolation of the smoothed ACF.

cAMP-sensitive HCN4 channel. In both nuclei investigated, TC neurons did not switch from burst to tonic firing in response to cAMP over a broad range of injected currents (Figure 4A, Figure 6A, and Supplemental Figure 7C). A possible explanation for this finding could be that HCN4 and HCN2 operate in different compartments of TC neurons. In support of this hypothesis, we found that HCN2 is enriched in somata compared with the dendrites, while HCN4 expression shows the opposite polarity (Figure 2). An alternative explanation could be that the residual cAMP-dependent $\mathrm{I}_{h}$ upregulation provided by HCN4 is not sufficient to rescue normal firing. Importantly, HCN2EA mice showed impaired visual learning, indicating that visual 
A
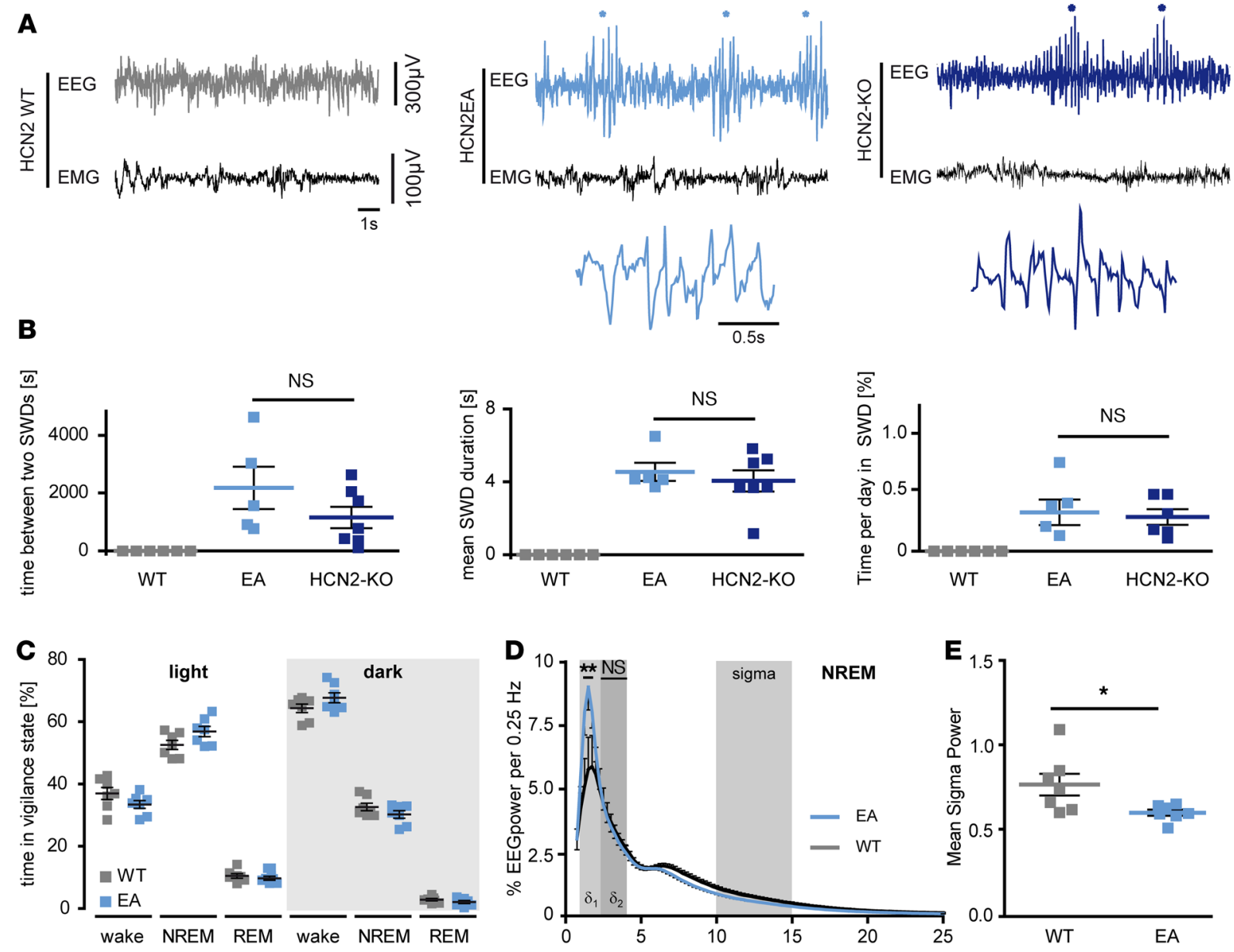

Figure 7. Absence epilepsy in HCN2EA mice. (A) EEG traces of WT (left), HCN2EA (middle), and global HCN2-KO mice (right panel). Spike and wave discharges (SWDs) in the EEG of HCN2EA and global HCN2-KO are marked by asterisks. SWDs concur with episodes of low activity in the EMG. Higher magnifications of SWD traces are displayed below the EEC of HCN2EA and HCN2-KO mice. Representative traces of animals analyzed in B. (B) SWD characteristics of HCN2EA (light blue, $n=5$ ) and global HCN2-KO (dark blue, $n=6$ ) animals compared with WT traces (gray, $n=6$ ) regarding the time between 2 SDWs (left), mean SWD duration (middle), and the time per day in SWDs (right). NS, not significant (1-way ANOVA, Bonferroni's post hoc test). (C) Time spent in different vigilance states (wake, NREM and REM sleep) during light and dark conditions (HCN2EA, blue; WT, gray; $n=7$ ). (D) Power spectra of NREM sleep in HCN2EA (blue, $n=7$ ) and WT animals (gray, $n=7$ ). ${ }^{*} P<0.01$ by 2 -way ANOVA. (E) Mean sigma power in HCN2EA (blue, $n=7$ ) and WT animals (gray, $n=7$ ). ${ }^{*} P=0.0175$ by Mann-Whitney test.

learning critically relies on CDR of firing modes in the dLGN. Increased burst firing of thalamocortical cells in the dLGN may lead to a disruption of the flow of visual information to the visual cortex, and therefore cause defects in thalamus-dependent visual behavior. Notably, the impairment in visual learning observed in HCN2EA could involve multiple neuronal circuits. Given that HCN2 is by far the predominant isoform in the dLGN, the likelihood that HCN2 in this nucleus is a major contributor to the effect is very high.

The occurrence of generalized seizures in HCN2EA mice provides a striking example of absence epilepsy of thalamic origin. HCN2EA mice display some phenotypes observed in global HCN2-KO mice including absence seizures, as characterized by SWDs in EEG recordings from HCN2EA mice that were indistinguishable from those found in global HCN2-KO mice. In addition, during episodes of SWDs mice displayed behavioral arrest (Supplemental Videos 1-3), a characteristic hallmark for absence seizures. However, other phenotypes are not present, including whole-body tremor, ataxia, lower body weight, and reduced life span (Supplemental Figure 2) $(8,9)$. These observations suggest that the loss of dynamic cAMP modulation does not completely impair HCN2 function in vivo. Rather, it has a specific impact on a subset of functions that reflects the activity of distinct neuronal circuits. This finding also supports previous studies performed in rat models of epilepsy (WAG/Rij and GAERS) in which an upregulation of HCN1 in thalamic TC neurons, and thereby reduced cAMP regulation, was proposed to 
A

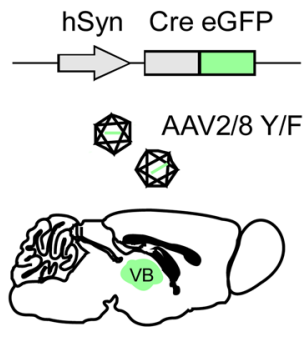

floxed mice

B
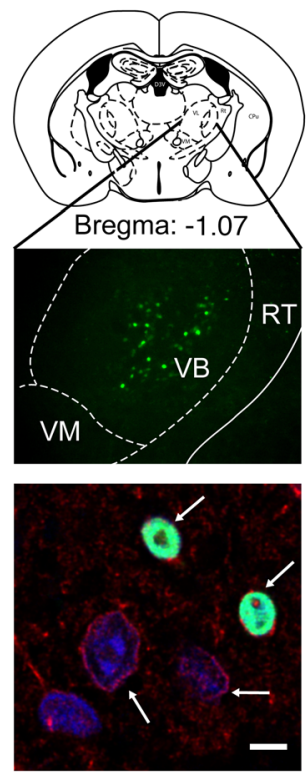

C
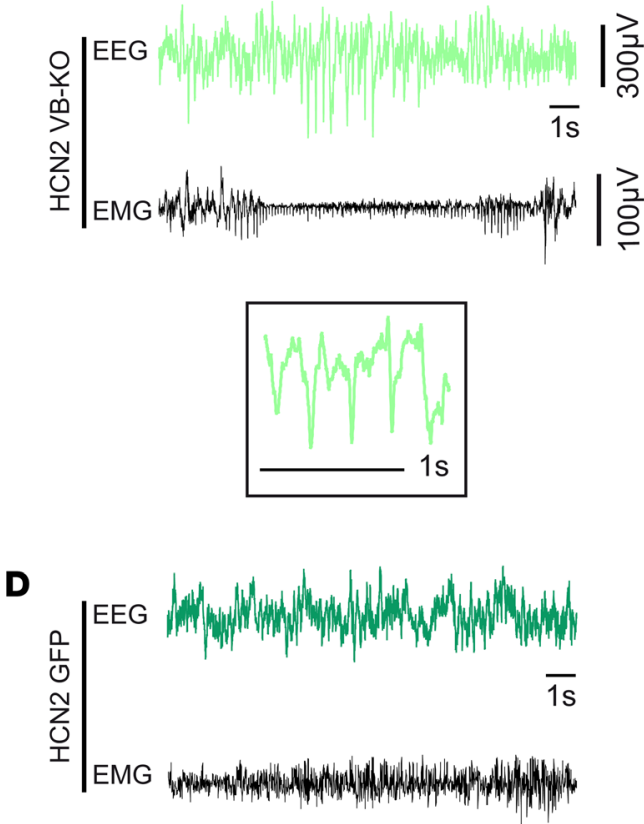

$\mathbf{E}$

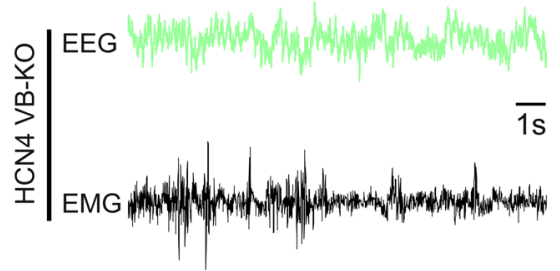

Figure 8. Absence epilepsy in VB-specific HCN2-KO mice. (A) Strategy for specific deletion of HCN channels in the VB. Left panel: Scheme of the AAV2/8-hSyn-Cre-EGFP vector that was injected stereotactically into the VB of $H C N^{f / / 7}$ mice. The schematic brain shows the injected region in the thalamus. Only cells in the VB show the green EGFP signal due to viral transfection. (B) Staining with anti-HCN2 antibody (red) in the VB region shows that Cre/EGFP-positive neurons (green) lack HCN2, while nontransduced (EGFP-negative) cells express HCN2 in the plasma membrane (marked by white arrows). Scale bar: $5 \mu \mathrm{m}$. VM, ventromedial region. (C) VB-specific HCN2-KO mice show SWDs (marked with an asterisk) in EEC traces (representative trace, $n=3$ ). The inset shows a magnification of the SWD. (D) Representative EEG (green) and EMG (black) traces of an $H C N 2^{f / f l}$ mouse that was injected with a control AAV vector that expresses only EGFP ( $n$ = 3). (D) Representative EEG (green) and EMG (black) traces of a VB-specific HCN4-KO animal that was generated by injecting the same vector as displayed in Figure 7A into the VB of an $\mathrm{HCN} 4^{f / / f l}$ mouse $(n=3)$.

be part of the pathomechanism leading to seizures $(29,42)$. Previous rodent models for absence epilepsy have focused on abnormal cortical activity and on a perturbation of cortically controlled feedforward inhibition of TC cells through the nRT $(43,44)$. Absence epilepsy was also found in animals deficient in corticoreticular excitation, which leads to a hyperexcitability of TC cells due to the lack of feedforward inhibition (45). Moreover, real-time induction of thalamic burst firing using optogenetics evoked absence seizures in animal models (46).

However, it is possible that other brain regions expressing HCN2 (e.g., the cortex) could also contribute to this phenotype (47). We sought to clarify this question by acutely deleting HCN2 locally in the VB of adult mice using an AAV-mediated approach, which was designed to avoid compensatory changes in gene expression pattern that could occur in classical knockouts and also allows for the spatial control of gene deletion. In agreement with a key role of the VB, these mice displayed SWDs in EEG recordings that were qualitatively identical to those observed in HCN2EA or global HCN2-KO mice. In line with the distinct functional profile of HCN4 discussed above, mice with a VB-specific deletion of HCN4 did not show SWDs. Taken together, these findings support the key role of HCN2 in information processing in the VB and suggest that HCN4 is involved in functions that are not directly coupled to those controlled by HCN2. 
Our findings on the level of the channel (voltage clamp), on the cellular level (current clamp), and systemic in vivo level are summarized in Supplemental Figure 7. The cartoon illustrates that a fixed amount of channel activity is present for a given membrane potential in the absence of cAMP dynamics (steady-state activation curve on the left [closed symbols] of WT HCN2 channels in the absence of cAMP). On top, channel activity of WT HCN2 can be gradually tuned according to cellular needs by cAMP via upstream cAMP-sensitive signaling effectors like $G_{\alpha S}$ (Supplemental Figure 7A; red area; HCN2 channels with cAMP dynamics). In contrast, HCN2EA channels cannot bind cAMP and hence, are unresponsive to changes in the cAMP concentration. The dynamic range for CAMP-sensitive tuning is represented by the red area in Supplemental Figure 7. Within the physiological range of membrane voltages, cAMP can recruit up to $300 \%$ of HCN2 channel activity, whereas in the absence of cAMP the current is greatly reduced (less than one-third).

In the thalamus, the transition between burst mode activity and the transmission mode is regulated by cAMP-sensitive upregulation of HCN2 channels. This is critically important for keeping neurons in the transmission mode during wakefulness and thereby protects the brain from absence epilepsy and behavioral arrest. First, TC neurons lacking cAMP modulation of HCN2 channels have a slightly hyperpolarized RMP, which facilitates the transition to the burst-firing mode. Consequently, in the semi-intact circuit TC cells tend to show synchronized low-frequency activity at their endogenous clock-like pace, as observed previously when blocking $I_{h}$ pharmacologically (26). This will in turn facilitate the excitation of reticular cells and create conditions that are prone to hypersynchrony in reticular-thalamic and cortico-thalamic network loops in vivo. Second, the absence of CDR of HCN2 may lead to an imbalance between depolarizing inward currents carried by $I_{h}$ and hyperpolarizing currents such as potassium currents. As a result, the membrane potential during the transmission mode (tonic firing) would be less stable and prone to perturbing influences. This hypothesis is consistent with a similar stabilizing role for HCN channels regarding the RMP in other systems $(48,49)$. In vivo, this excitatory-inhibitory imbalance would manifest on the behavioral level as sleep-like episodes, behavioral arrest, spike and wave discharges in EEG recordings, as well as episodes of behavioral arrest, all of which are consistent with the clinical symptoms of absence episodes. The transition between sleep and waking is accompanied by marked changes in the activity of forebrain and brainstem arousal systems. In these arousal-promoting mechanisms, neurotransmitters are released that lead to cAMP upregulation of $I_{h}$, membrane depolarization, and promotion of tonic firing in thalamic cells (27). However, in spite of the pronounced deficiency of cAMP signaling, animals spent comparable time in wakefulness. Although these data argue against a major role of CDR of HCN2 in the control of waking from sleep during arousal, it should be noted that arousal promotion also includes cAMP-independent neurotransmitter signaling, such as $\mathrm{G}$ protein-mediated closure of $\mathrm{K}^{+}$channels to depolarize TC cells $(50,51)$. The redundancy of wake-promoting mechanisms could therefore overcome the deficiency in CDR of HCN2.

Thalamic HCN channel-dependent activities have also been implicated in the control of low-frequency sleep waves, particularly in the sigma $(10-15 \mathrm{~Hz})$ and delta $(0.5-4 \mathrm{~Hz})$ frequency range (1). The decrease in sigma power noted in the NREM sleep spectral profile is in line with the notion that HCN channels are relevant for sleep spindle generation $(23,52)$. The augmentation of power in the slow delta range $(1-2.5$ $\mathrm{Hz}$ ) in $\mathrm{HCN} 2 \mathrm{EA}$ animals suggests that thalamic activity regulates low-frequency oscillatory activity in the delta band and indicates a complex interplay of thalamic and cortical rhythm generators in the composition of the delta band, which is a major frequency band of NREM sleep. Our observations are in line with increasing evidence showing that slow and fast delta-frequency components serve different roles in homeostatic sleep regulation (53). Furthermore, the mouse model generated in this study will be a valuable tool to examine the contribution of HCN2 in other systems in which CDR of $\mathrm{I}_{h}$ has been proposed.

Our findings on HCN2-cAMP-dependent burst control and absence epilepsy are thus fully consistent with a central role of thalamic burst propensity in thalamic epileptogenesis. In addition, we show that HCN2 channels are a molecular target in which burst firing of TC cell activity can be regulated, thereby expanding the range of proposed anti-absence drug strategies.

\section{Methods}

PyMOL. The figure of the CNBD structure was prepared using PyMOL Molecular Graphic System, version 1.3 (Schrodinger LLC). The structure is based on the sequence 483-607 of the human HCN1 structure in complex with cAMP (15), downloaded from the RCSB protein database (https://www.rcsb.org/, accession code $5 \mathrm{U} 6 \mathrm{P})$. 
Generation of HCN2EA mice. The targeting construct was amplified using a BAC clone (Genome Systems) isolated from a genomic 129/SvJ library. Base pairs in exon 7 of murine HCN2 gene coding for amino acids R591 and T592 of mHCN2 were mutated to E591 and A592 by site-directed mutagenesis [(CGT) R591E(GAA)/(ACG)T592A(GCG)]using a QuikChange II XL site-directed mutagenesis kit (Agilent) according to the manufacturer's protocol. For embryonic stem cell selection, an ACN-selection cassette (54) flanked by loxP sites was inserted in intron 5 of the targeting construct. The construct was electroporated into R1 embryonic stem cells, and G418-resistant clones were screened by Southern blot analysis. Correctly targeted clones were injected into C57BL/ $6 \mathrm{~N}$ blastocysts, and the resulting chimeric mice were mated with C57BL/6N mice for germline transmission. Primers for genotyping the offspring were $5^{\prime}$-AGTTGTACTCAACCAGTGGC-3' (sense) and 5'-TAGTCACGGTCACTGCCAAG-3' (antisense). The correct integration of the mutated base pairs was verified by amplifying exon 7 (sense: 5'-GGAAGAAGATGTACTTCATCCAG-3'; antisense: 5'-TGCCCCACTGGAATGGAGCC-3') and subsequent sequencing (Eurofins).

Immunohistochemistry. Adult mice were sacrificed and brains were deep-frozen in isobutane. Immunohistochemistry was performed in $12-\mu \mathrm{m}$-thick cryosections as described previously (55). The following primary antibodies were used: anti-HCN1 (1:300, Alomone, APC-056), anti-HCN2 (1:300, Alomone, APC-030), anti-HCN3 (1:300, Alomone, APC-057), anti-HCN4 (1:300, Alomone, APC-052), Cy3 donkey anti-rabbit IgG (711-165-152, Jackson ImmunoResearch), or Alexa488 donkey anti-rabbit IgG (711-545152 , Jackson ImmunoResearch). Images were acquired at a minimum of $3208 \times 3208$ pixel resolution in an inverted Leica TCS-SP8 confocal microscope, 10-40×, 1.4-NA objective and $600 \mathrm{~Hz}$. For the comparison of WT and HCN2EA littermates, slices were processed using the same protocol on the same day. The acquisition was performed with identical laser intensities and scanner settings. The intensities of the HCN fluorescence were analyzed using the plot profile tool of ImageJ software (56).

Primary neurons. Primary neurons from WT and HCN2EA mice were isolated as described previously and transfected with either HCN2 (mHCN2_pcDNA3) or HCN2EA DNA (mHCN2EA_pcDNA3) using high-calcium phosphate transfection on DIV4 (57). Immunocytochemistry was performed on DIV14 as described (57). The following primary antibodies were used: anti-HCN2 (1:300, Alomone, APC-030), antiMAP2 (1:500, Sigma-Aldrich, M9942) followed by Cy3 donkey anti-rabbit IgG (1:400, Jackson ImmunoResearch, 711-165-152) or DyLight 488 anti-mouse (1:800, Jackson ImmunoResearch, 715-485-151).

Western blot. Samples from the dLGN and the VB were punched from adult mouse brain and homogenized in RIPA buffer containing $50 \mathrm{mM}$ Tris $\mathrm{pH}$ 8.0, $150 \mathrm{mM} \mathrm{NaCl}, 1 \%$ Triton X-100, 0.5\% sodium deoxycholate, $0.1 \%$ SDS, and Protease Inhibitor Cocktail (Complete, Mini, Roche). The preparation of brain membranes has been described previously (58). Western blotting was performed using standard methods. Primary antibodies used were anti-HCN2 (1:1000, Alomone, APC-030), anti-HCN4 (1:1000, Alomone, APC-052), anti-ATP1A1 (1:1000, a6F, DSHB, University of Iowa), and anti-Trip8b (1:1000, N212/7, Neuromab). Secondary horseradish-peroxidase-conjugated antibodies were used (rabbit: 1:1000, sc-2030; mouse: 1:2000, sc-2031; Santa Cruz Biotechnology). Signals were acquired with a luminescence image analyzer (ChemiDoc MP; Bio-Rad) and quantified using ImageLab software (Bio-Rad).

Coimmunoprecipitation. Co-IPs were performed with anti-HCN2 antibody (Alomone, APC-030), antiHCN4 (Alomone, APC-052), or anti-Trip8b (N212/7, Neuromab) using Novex Protein G magnetic beads (Thermo Fisher Scientific) according to the manufacturer's protocol.

$q R T-P C R$. The VB and dLGN regions of 4- to 5-month-old mice were dissected in $400-\mu \mathrm{m}$ coronal slices (Vibratome; Leica VT1200S). RNA was isolated using the RNeasy Micro Kit (Qiagen). cDNA was synthesized with the RevertAid First Strand cDNA Synthesis Kit (Thermo Fisher Scientific). qRT-PCR was performed using the following primer sequences: HCN1 for: CTGCTGCAGGACTTCCCACCA, HCN1 rev: ATGCTGACAGGGGCTTGGGC, HCN2 for: CAGGAACGCGTGAAGTCGGCG, HCN2 rev: TCCAGGGCGCGGTGGTCTCG, HCN3 for: TGGCCATGGACCGGCTTCGG, HCN3 rev: GAGCCAGGCCCCGAACACCAC, HCN4 for: AGGGCCTTCGAGACGGTTGCGC, HCN4 rev: GGCCATCTCACGGTCATGCCG, ALAS for: TCGCCGATGCCCATTCTTATC, ALAS rev: GGCCCCAACTTCCATCATCT.

rAAV preparation and stereotaxic injections. Recombinant AAV particles (pAAV-hSyn-Cre-p2A-EGFP or pAAV-hSyn-EGFP) were produced with a $2 / 8 \mathrm{Y} / \mathrm{F}$ capsid variant as described previously (59). Viral particles $\left(1 \times 10^{10}\right)$ were delivered bilaterally to the VB region of the thalamus of adult $(\mathrm{P} 60-\mathrm{P} 70) \mathrm{HCN} 2 \mathrm{~L} 2$ or HCN4 L2 mice anesthetized with isoflurane by stereotaxic injection using a 34-G Hamilton syringe. The stereotaxic coordinates for bilateral targeting were as follows: A/P, $-1.2 ; \mathrm{M} / \mathrm{L}, \pm 1.7 ; \mathrm{D} / \mathrm{V},-3.2$; and $\mathrm{A} / \mathrm{P}$, -3.2 (from bregma). Volume $(0.5 \mu \mathrm{l}$ at each injection site) and speed $(100 \mathrm{nl} / \mathrm{min})$ of the injections were 
controlled by a WPI Micro4 pump. The Hamilton syringe was retracted from the brain after a 3-minute waiting period to allow diffusion to occur. After suturing and disinfecting, mice were allowed to recover for 2 weeks prior to the start of the in vivo EEG experiments.

Telemetric EEG measurements. To measure the EEG and EMG traces, a telemetric EEG transmitter (F20EET, Data Science International) was implanted using the following procedure. The head of the mouse was fixed in a stereotactic apparatus and the body temperature was maintained constant at $37^{\circ} \mathrm{C}$ via a rectal feedback control system. By a small (1.5-cm-long) incision, the scalp was first opened along the median of the head and the skullcap (cranium cerebrale) was dissected. A second incision was made caudally between the scapulae along the longitudinal axis. Using blunt scissors, a small pocket was opened for the transmitter and the 2 electrodes for electromyography (EMG) were sutured on the shoulder muscle. Two tiny holes were drilled into the skullcap ( $0.7 \mathrm{~mm}$ in diameter) using the following coordinates: A/P, $-2.0 ; \mathrm{M} / \mathrm{L}, 1.8$ (from bregma; recording electrode) and $\mathrm{A} / \mathrm{P},-1.0 ; \mathrm{M} / \mathrm{L},-0.5$ (from lambda; reference electrode). After suturing and disinfection, mice were allowed to recover for 2 weeks before in vivo EEG experiments.

Cortical electroencephalogram (EEG/ECoG) and EMG traces of freely moving mice were recorded using A.R.T. software (Data Science International) and videotaped. EMG traces were high-pass filtered at $10 \mathrm{~Hz}$. The vigilance states (wake, NREM sleep, and REM sleep) were scored using Neuroscore3.2.1 (Data Science International). The EEG traces were Fourier transformed (DFT) in a frequency range from 0 to $26 \mathrm{~Hz}$ and an epoch size of 4 seconds for NREM sleep using a Hamming Window. Spike trains (spike and wave discharges, SWDs) were defined as a minimum number of 10 spikes with a maximal spike interval of 0.6 seconds.

Behavioral analysis. Behavioral studies were performed with male adult mutant mice and WT littermates. Mice were housed in standard cages in a 12-hour light/12-hour dark cycle. Water and rodent chow were available ad libitum during all tests. All tests were performed during the light phase of the light/ dark cycle by trained observer blind to genotype. The virtual-cliff test and the Morris water maze were videotaped using a computer-assisted data acquisition system (VideoMot, TSE). The experiments were performed and analyzed blindly with respect to the animals' genotype.

Footprint analysis. Mice were trained to walk on a sheet of blank paper along a runway $(7 \times 30 \mathrm{~cm})$. Their front and hind paws were painted with nontoxic paint in blue and green colors, respectively. The resulting footprint traces were analyzed by measuring the following morphometric parameters: stride length, hind base width (normalized to the body length), and hindpaw angle.

Visual discrimination task. The procedure is a test for discrimination of visual stimuli, processed by the geniculate pathway in the dLGN of the thalamus $(60,61)$. Briefly, mice were trained to discriminate between a conditioned stimulus $\left(\mathrm{CS}^{+}\right)$that is connected to a hidden platform in a water maze and nonconditioned stimulus $\left(\mathrm{CS}^{-}\right)$images. The $\mathrm{CS}^{+}$was connected to a hidden platform at the open side of a trapezoid-shaped swimming pool. Each mouse was given 3 blocks of 10 training units per day in 4 consecutive days. One training unit consisted of no more than 5 trials for swimming consecutively into the wrong arm. One training unit was terminated once a mouse crossed the choice line and climbed onto the platform. To avoid the effect of positional learning, the side of the $\mathrm{CS}^{+}$image and the platform were pseudorandomly changed after each trial using a Gellerman schedule (LRLLRLRR; $L=1$ eft, $\mathrm{R}=$ right). To assess the visual discrimination capacity, the mean probability of a correct choice per day was analyzed.

Virtual-cliff test. The open field consisted of a white acrylic glass arena of $48 \times 48 \times 30 \mathrm{~cm}$ under uniform (25 lux) lightning condition. Mice were allowed to explore the arena for 10 minutes. The arena was subdivided into 2 zones using the SMART software: zone 1, the central square area of $25 \times 25 \mathrm{~cm}$ equidistant from the walls; and zone 2 , the remaining borders. The transitions between the 2 zones were monitored.

Morris water maze. The Morris water maze is a test designed for hippocampus-dependent learning (62). Briefly, mice were trained for 4 days ( 8 trials per day) to locate a stable platform (10 $\mathrm{cm}$ in diameter). The platform was placed in a circular swimming pool $(120 \mathrm{~cm}$ in diameter, $70 \mathrm{~cm}$ high, white plastic) filled with water. The starting position of the mouse was changed from trial to trial in a pseudorandom order, whereas the platform was kept in a constant location. Distal cues in the testing room and the water maze, such as patterned cardboards, were provided as spatial references. Trials were terminated if the mouse had climbed onto the platform or after swimming for 2 minutes.

Acute thalamic slice patch recordings. Horizontal or coronal slices $(250-300 \mu \mathrm{m})$ containing the VB complex or the dLGN, respectively, were prepared on a vibratome (HM 650V, Microme) in an ice-cold oxygenated $\left(95 \% \mathrm{O}_{2}, 5 \% \mathrm{CO}_{2}\right)$ solution containing $(\mathrm{mM}): 65.5 \mathrm{NaCl}, 2.5 \mathrm{KCl}, 1.25 \mathrm{NaH}_{2} \mathrm{PO}_{4}, 26 \mathrm{NaHCO}_{3}, 0.5 \mathrm{CaCl}_{2}$, $7 \mathrm{MgCl}_{2}, 105$ sucrose, 24.7 glucose, and 1.7 ascorbic acid. Slices were incubated at $35^{\circ} \mathrm{C}$ for 30 minutes and 
left at room temperature in an oxygenated artificial cerebrospinal fluid (ACSF) solution containing (mM): $131 \mathrm{NaCl}, 2.5 \mathrm{KCl}, 1.25 \mathrm{NaH}_{2} \mathrm{PO}_{4}, 26 \mathrm{NaHCO}_{3}, 2 \mathrm{CaCl}_{2}, 1.2 \mathrm{MgCl}_{2}, 18$ glucose, and additionally with 1.7 ascorbic acid for storage until recording.

Whole-cell recordings were obtained from TC neurons of the VB or dLGN in slices perfused at $31^{\circ} \mathrm{C}-33^{\circ} \mathrm{C}$ with oxygenated ACSF. TC neurons were visualized with an upright microscope (Axioskop 2 FS plus, Zeiss) with an IR CCD camera (VX55, Till Photonics). A horizontal puller (DMZ Universal Electrode Puller, Zeitz-Instruments) was used to pull patch pipettes from borosilicate glass tubing (GC150TF-10, Harvard Apparatus). Patch pipettes were filled with the following solution (mM): $140 \mathrm{KMeSO}_{4}, 10 \mathrm{HEPES}$, $10 \mathrm{KCl}, 0.1 \mathrm{EGTA}, 10$ phosphocreatine, and $4 \mathrm{MgATP}$. The osmolarity was adjusted to $305 \mathrm{mOsm} / 1$, and the $\mathrm{pH}$ was adjusted to 7.3. In addition, $0.2 \mathrm{mM}$ GTP was added freshly before each experiment. For experiments including cAMP, $1 \mu \mathrm{M}$ 8-bromo cAMP (Sigma-Aldrich, B7880) was added to the pipette solution. The resistance of the electrode was 2.5-6.0 $\mathrm{M} \Omega$. A liquid junction potential of $-8.9 \mathrm{mV}$ was calculated using the Junction Potential tool of the Clampex 10.5 software and corrected off-line (63).

Voltage clamp recordings. For measurement of $\mathrm{I}_{\mathrm{h}}$, slices were perfused with an oxygenated ACSF plus 1 $\mathrm{mM} \mathrm{BaCl}_{2}$ and $0.5 \mu \mathrm{M}$ tetrodotoxin.

To determine $I_{h}$ steady-state activation curves, TC neurons were clamped at a holding potential of -45 $\mathrm{mV}$ from which pulses of 2.5 -second duration were applied from $-140 \mathrm{mV}$ to $-30 \mathrm{mV}$ in $10-\mathrm{mV}$ increments, followed by a $250-\mathrm{ms}$ test pulse to $-140 \mathrm{mV}$. Tail currents measured immediately after the final step to -140 $\mathrm{mV}$ were normalized to the maximal current $\left(\mathrm{I}_{\max }\right)$ and plotted as a function of the preceding membrane potential (10). The curves were fitted with a Boltzmann function: $\mathrm{I} / \mathrm{I}_{\max }=(\mathrm{A} 1-\mathrm{A} 2) /\left(1+\mathrm{e}^{\left[\mathrm{V}-\mathrm{v}_{0.5}{ }^{] / k}\right.}\right)+\mathrm{A} 2$, where $\mathrm{V}_{0.5}$ and $k$ represent the half-maximal voltage and Boltzmann slope factor, respectively, and $\mathrm{A} 1$ and A2 represent the initial and final I/I $\mathrm{I}_{\max }$ values. The current density was calculated as the amplitude recorded at $-140 \mathrm{mV}$ divided by the cell capacitance. Time constants were fitted by a single exponential function.

Current-clamp recordings. To record firing patterns, slices were perfused with an oxygenated ACSF. TCs were clamped at their RMP and 300-ms depolarizing pulses ranging from 50 to $400 \mathrm{pA}$ were applied. To test for the influence of the membrane potential on firing modes, TCs were first clamped at their RMP and 300 -ms depolarizing pulses from 50 to $200 \mathrm{pA}$ were applied. Next, by application of constant currents ranging from -15 to $-30 \mathrm{pA}$ or from +10 to $+30 \mathrm{pA}$, TCs were brought to a more hyperpolarized or depolarized potential and experiments were repeated. The voltage sag was determined by negative-current injections for 2.5 seconds in $20-\mathrm{pA}$ increments from -100 to $-20 \mathrm{pA}$. The sag ratio was calculated by dividing the steady state of the sag measurement by the peak voltage deflection (64).

Cell culture and heterologous expression. HEK293T cells (ATCC, CRL-3216) were maintained in DMEM medium (Gibco, Life Technologies, Inc.) supplemented with 10\% fetal bovine serum, $100 \mathrm{units} / \mathrm{ml}$ penicillin, and $100 \mu \mathrm{g} / \mathrm{ml}$ streptomycin, and incubated at $37^{\circ} \mathrm{C}$ with $10 \% \mathrm{CO}_{2}$. At $80 \%$ confluence, HEK293T cells were seeded on 6-well plates (diameter $3.5 \mathrm{~cm}$ ) for transfection. After 24 hours, cells were transfected with expression plasmid DNA ( $4 \mu \mathrm{g}$ of each plasmid per well) using the Turbofect transfection reagent (Thermo Fisher Scientific). For electrophysiological measurements, transfected cells were detached using $0.05 \%$ trypsin-EDTA (Gibco, Life Technologies, Inc.) and replated on to 12-mm poly-L-lysine-coated coverslips in 24-well plates.

Stable HCN2 or HCN2EA HEK293 cells were generated using the Flp-In-293 Cell Line (Thermo Fisher Scientific).

Electrophysiology of HEK cells. Currents of heterologously expressed HCN2 and HCN2EA channels were measured using the whole-cell patch-clamp technique as previously described with slight changes (65). The extracellular solution was composed of (mM): $135 \mathrm{NaCl}, 0.5 \mathrm{MgCl}_{2}, 1.8 \mathrm{CaCl}_{2}, 5 \mathrm{HEPES}, 5$ $\mathrm{KCl}, \mathrm{pH} 7.4$ adjusted with $\mathrm{NaOH}$. The effect of cAMP was tested for by adding $100 \mu \mathrm{M}$ cAMP to the intracellular solution the day of recording. Steady-state activation curves were determined by applying a hyperpolarizing voltage of $-130 \mathrm{mV}$ from a holding potential of $-40 \mathrm{mV}$ for 2 seconds (for both WT and HCN2EA). The amplitude of $I_{h}$ was calculated by subtracting the instantaneous current amplitude from the steady-state current. The density of $I_{h}$ was calculated by dividing the $I_{h}$ current amplitude at $-130 \mathrm{mV}$ by the membrane capacitance obtained during whole-cell recordings.

Internal perfusion experiments. Planar patch-clamp technology combined with a fast internal perfusion system (Port-a-Patch, Nanion Technologies) was used to examine the kinetics and current density of HCN2 and HCN2EA channels in stably transfected HEK293 cells. Data were digitized at $20 \mathrm{kHz}$ and filtered at $2.9 \mathrm{kHz}$. Fast and slow capacitive transients were cancelled by the compensation circuit of the EPC-10 double amplifier. All recordings were obtained at room temperature. The extracellular solution was composed of (mM): 110 
$\mathrm{NaCl}, 5 \mathrm{KCl}, 1.8 \mathrm{CaCl}_{2}, 0.5 \mathrm{MgCl}_{2}$ and $5 \mathrm{HEPES}$ ( $\mathrm{pH}$ adjusted to 7.4 with $\mathrm{NaOH}$ ). The intracellular solution contained (mM): $70 \mathrm{KCl}, 60 \mathrm{KF}, 10 \mathrm{NaCl}, 0.5 \mathrm{MgCl}_{2}$ and 5 HEPES (pH adjusted to 7.4 with $\mathrm{KOH}$ ). All solutions were sterile filtered before use. Currents were evoked from a holding potential of $-40 \mathrm{mV}$ by applying 2-second pulses of $-150 \mathrm{mV}$ every 10 seconds. The effect of $10 \mu \mathrm{M}$ cAMP was examined by exchanging the standard internal solution with internal solution supplemented with cAMP using the internal perfusion system after the cells reached steady state after rundown.

Two-photon calcium imaging. Experiments were carried out in horizontal brain slices as described previously $(8,66-68)$. We guided a micropipette with a resistance of 3 to $5 \mathrm{M} \Omega$ to the VB region in the thalamus and applied pressure (10 psi) for 2-4 minutes to eject the solution from the pipette. Activity of thalamic neurons was monitored by imaging the fluorescence changes with a custom-built 2-photon microscope based on a Ti:sapphire pulsing laser operating at a wavelength of $800 \mathrm{~nm}$ and resonant galvo-mirror system ( 8 or $12 \mathrm{kHz}, \mathrm{GSI}$ ) through a $40 \times, 0.8$ numerical aperture (Nikon) water immersion objective. Full-frame images were acquired at $40 \mathrm{~Hz}$ using custom-written software based on LabView (National Instruments). We performed image analysis offline. Regions of interest (ROIs) were drawn around individual somata, and then relative fluorescence change $(\Delta \mathrm{F} / \mathrm{F})$ versus time traces were generated for each ROI. Based on the original fluorescence recordings, binary traces were generated in which time points of fluorescence peaks were assigned the value " 1 " in contrast to " 0 " for all other time points. A cubic spline interpolation was performed on smoothed autocorrellograms that were calculated for 120-second-long binary traces using Igor Pro software (Wavemetrics). A regular pattern of satellite peaks revealed oscillatory activity.

Statistics. Electrophysiology data were analyzed using PatchMaster software (HEKA Elektronik, Harvard Bioscience). Data plotting and statistical analysis were performed using GraphPad Prism 5. All values are presented as mean \pm SEM for the indicated number $n$ of experiments. $P$ values were calculated by Mann-Whitney test or 1- or 2-way analysis of variance (ANOVA) with Bonferroni's post hoc test. ${ }^{*} P<$ $0.05,{ }^{* *} P<0.01$, and ${ }^{* * *} P<0.001$ were considered statistically significant.

Study approval. The studies were carried out in accordance with the approved guidelines of the local committee of laboratory animal care (District Government of Upper Bavaria) and German Laws on animal welfare (Tierschutzgesetz). The HCN2-KO and HCN2 L2 mouse lines have already been published (8) as well as the $\mathrm{Cnga3}^{-1-} \mathrm{Rho}^{-/-} \mathrm{Opn4^{-/- }}$ triple-KO mouse (36).

\section{Author contributions}

VH designed and performed behavioral experiments, viral knockdown, immunohistochemistry, Western blots, EEG measurements, generated HCN2EA expression vectors and the HEK293 stable HCN2EA cell line, and analyzed data. SS performed EEG measurements and analyzed sleep parameters. MSS, HH, and KLM performed electrophysiological experiments in thalamic slices. JK, RDR, and SF performed electrophysiological experiments in HEK293 cells. M. Brümmer performed qRT-PCR analysis. CG generated the HCN2EA mouse together with MM. A. Ludwig generated the floxed HCN2 and HCN4 mice. JH, BZ, and AK performed calcium imaging in thalamic slices. DI helped to implement telemetric EEG recordings. A. Lüthi helped to implement electrophysiology in thalamic slice preparations. CWS and M. Biel designed experiments and analyzed data. CWS and M. Biel wrote the manuscript.

\section{Acknowledgments}

We thank Mario R. Capecchi, Department of Human Genetics and Howard Hughes Medical Institute, University of Utah School of Medicine, Salt Lake City, USA for providing the ACN cassette. This work was supported by the Deutsche Forschungsgemeinschaft SFB870, projects B05 (to CWS), B10 (to M. Biel), and $\mathrm{B} 18$ (to $\mathrm{AK}$ ).

Address correspondence to: Martin Biel, Department of Pharmacy - Ludwig-Maximilians-Universität München, Butenandtstrasse 5-13, D 81377 München, Germany. Phone: 49.892.1807.7328; Email: martin.biel@1mu.de (M. Biel). Or to: Christian Wahl-Schott, Institut für Neurophysiologie - Medizinische Hochschule Hannover (MHH), Carl-Neuberg-Str. 1, 30625 Hannover, Germany, Email: christian. wahl@cup.uni-muenchen.de or Wahl-Schott.Christian@mh-hannover.de (C. Wahl-Schott). 
1. Biel M, Wahl-Schott C, Michalakis S, Zong X. Hyperpolarization-activated cation channels: from genes to function. Physiol Rev. 2009;89(3):847-885.

2. Robinson RB, Siegelbaum SA. Hyperpolarization-activated cation currents: from molecules to physiological function. Annu Rev Physiol. 2003;65:453-480.

3. Notomi T, Shigemoto R. Immunohistochemical localization of Ih channel subunits, HCN1-4, in the rat brain. J Comp Neurol. 2004;471(3):241-276.

4. Moosmang S, Biel M, Hofmann F, Ludwig A. Differential distribution of four hyperpolarization-activated cation channels in mouse brain. Biol Chem. 1999;380(7-8):975-980.

5. Nolan MF, et al. The hyperpolarization-activated HCN1 channel is important for motor learning and neuronal integration by cerebellar Purkinje cells. Cell. 2003;115(5):551-564.

6. Nolan MF, et al. A behavioral role for dendritic integration: HCN1 channels constrain spatial memory and plasticity at inputs to distal dendrites of CA1 pyramidal neurons. Cell. 2004;119(5):719-732.

7. Thuault SJ, et al. Prefrontal cortex HCN1 channels enable intrinsic persistent neural firing and executive memory function. J Neurosci. 2013;33(34):13583-13599.

8. Ludwig A, et al. Absence epilepsy and sinus dysrhythmia in mice lacking the pacemaker channel HCN2. EMBO J. 2003;22(2):216-224.

9. Chung WK, et al. Absence epilepsy in apathetic, a spontaneous mutant mouse lacking the h channel subunit, HCN2. Neurobiol Dis. 2009;33(3):499-508.

10. Fenske S, et al. HCN3 contributes to the ventricular action potential waveform in the murine heart. Circ Res. 2011;109(9):1015-1023.

11. Stieglitz MS, et al. Disturbed processing of contextual information in HCN3 channel deficient mice. Front Mol Neurosci. 2017;10:436.

12. Stieber J, et al. The hyperpolarization-activated channel HCN4 is required for the generation of pacemaker action potentials in the embryonic heart. Proc Natl Acad Sci USA. 2003;100(25):15235-15240.

13. Pedarzani P, Storm JF. Protein kinase A-independent modulation of ion channels in the brain by cyclic AMP. Proc Natl Acad Sci USA. 1995;92(25):11716-11720.

14. Zagotta WN, Olivier NB, Black KD, Young EC, Olson R, Gouaux E. Structural basis for modulation and agonist specificity of HCN pacemaker channels. Nature. 2003;425(6954):200-205.

15. Lee CH, MacKinnon R. Structures of the human HCN1 hyperpolarization-activated channel. Cell. 2017;168(1-2):111-120.e11.

16. DiFrancesco D. The role of the funny current in pacemaker activity. Circ Res. 2010;106(3):434-446.

17. Pedarzani P, Storm JF. Dopamine modulates the slow $\mathrm{Ca}(2+)$-activated $\mathrm{K}^{+}$current IAHP via cyclic AMP-dependent protein kinase in hippocampal neurons. J Neurophysiol. 1995;74(6):2749-2753.

18. Emery EC, Young GT, Berrocoso EM, Chen L, McNaughton PA. HCN2 ion channels play a central role in inflammatory and neuropathic pain. Science. 2011;333(6048):1462-1466.

19. Heys JG, Hasselmo ME. Neuromodulation of I(h) in layer II medial entorhinal cortex stellate cells: a voltage-clamp study. J Neurosci. 2012;32(26):9066-9072.

20. Wang M, et al. Alpha2A-adrenoceptors strengthen working memory networks by inhibiting cAMP-HCN channel signaling in prefrontal cortex. Cell. 2007;129(2):397-410.

21. Shaikh AG, Finlayson PG. Excitability of auditory brainstem neurons, in vivo, is increased by cyclic-AMP. Hear Res. 2005;201(1-2):70-80.

22. Yamada R, Kuba H, Ishii TM, Ohmori H. Hyperpolarization-activated cyclic nucleotide-gated cation channels regulate auditory coincidence detection in nucleus laminaris of the chick. J Neurosci. 2005;25(39):8867-8877.

23. Lüthi A, McCormick DA. Modulation of a pacemaker current through $\mathrm{Ca}(2+)$-induced stimulation of cAMP production. Nat Neurosci. 1999;2(7):634-641.

24. Frère SG, Lüthi A. Pacemaker channels in mouse thalamocortical neurones are regulated by distinct pathways of cAMP synthesis. J Physiol (Lond). 2004;554(Pt 1):111-125.

25. McCormick DA. Neurotransmitter actions in the thalamus and cerebral cortex and their role in neuromodulation of thalamocortical activity. Prog Neurobiol. 1992;39(4):337-388.

26. McCormick DA, Pape HC. Properties of a hyperpolarization-activated cation current and its role in rhythmic oscillation in thalamic relay neurones. J Physiol (Lond). 1990;431:291-318.

27. McCormick DA, Bal T. Sleep and arousal: thalamocortical mechanisms. Annu Rev Neurosci. 1997;20:185-215.

28. Llinás RR, Steriade M. Bursting of thalamic neurons and states of vigilance. J Neurophysiol. 2006;95(6):3297-3308.

29. Budde T, et al. Impaired regulation of thalamic pacemaker channels through an imbalance of subunit expression in absence epilepsy. J Neurosci. 2005;25(43):9871-9882.

30. Liu DT, Tibbs GR, Paoletti P, Siegelbaum SA. Constraining ligand-binding site stoichiometry suggests that a cyclic nucleotide-gated channel is composed of two functional dimers. Neuron. 1998;21(1):235-248.

31. Chen S, Wang J, Siegelbaum SA. Properties of hyperpolarization-activated pacemaker current defined by coassembly of HCN1 and HCN2 subunits and basal modulation by cyclic nucleotide. J Gen Physiol. 2001;117(5):491-504

32. Santoro B, Wainger BJ, Siegelbaum SA. Regulation of HCN channel surface expression by a novel C-terminal protein-protein interaction. J Neurosci. 2004;24(47):10750-10762.

33. Zolles G, et al. Association with the auxiliary subunit PEX5R/Trip8b controls responsiveness of HCN channels to cAMP and adrenergic stimulation. Neuron. 2009;62(6):814-825.

34. Niell CM, Stryker MP. Modulation of visual responses by behavioral state in mouse visual cortex. Neuron. 2010;65(4):472-479.

35. Linden ML, Heynen AJ, Haslinger RH, Bear MF. Thalamic activity that drives visual cortical plasticity. Nat Neurosci. 2009;12(4):390-392.

36. Laprell $\mathrm{L}$, et al. Photopharmacological control of bipolar cells restores visual function in blind mice. J Clin Invest. 2017;127(7):2598-2611.

37. Huguenard JR, Prince DA. Intrathalamic rhythmicity studied in vitro: nominal T-current modulation causes robust antioscillatory effects. J Neurosci. 1994;14(9):5485-5502. 
38. Leresche N, Lightowler S, Soltesz I, Jassik-Gerschenfeld D, Crunelli V. Low-frequency oscillatory activities intrinsic to rat and cat thalamocortical cells. J Physiol (Lond). 1991;441:155-174.

39. Jacobsen RB, Ulrich D, Huguenard JR. GABA(B) and NMDA receptors contribute to spindle-like oscillations in rat thalamus in vitro. J Neurophysiol. 2001;86(3):1365-1375.

40. Astori S, et al. The $\mathrm{Ca}(\mathrm{V}) 3.3$ calcium channel is the major sleep spindle pacemaker in thalamus. Proc Natl Acad Sci USA 2011;108(33):13823-13828.

41. Saponaro A, et al. A synthetic peptide that prevents cAMP regulation in mammalian hyperpolarization-activated cyclic nucleotide-gated (HCN) channels. Elife. 2018;7:null.

42. Kuisle M, et al. Functional stabilization of weakened thalamic pacemaker channel regulation in rat absence epilepsy. $J$ Physiol (Lond). 2006;575(Pt 1):83-100.

43. Paz JT, Huguenard JR. Microcircuits and their interactions in epilepsy: is the focus out of focus? Nat Neurosci. 2015;18(3):351-359.

44. David F, et al. Suppression of hyperpolarization-activated cyclic nucleotide-gated channel function in thalamocortical neurons prevents genetically determined and pharmacologically induced absence seizures. J Neurosci. 2018;38(30):6615-6627.

45. Paz JT, et al. A new mode of corticothalamic transmission revealed in the Gria4(-/-) model of absence epilepsy. Nat Neurosci. 2011;14(9):1167-1173.

46. Sorokin JM, et al. Bidirectional control of generalized epilepsy networks via rapid real-time switching of firing mode. Neuron. 2017;93(1):194-210.

47. Stroh A, et al. Making waves: initiation and propagation of corticothalamic $\mathrm{Ca}^{2+}$ waves in vivo. Neuron. 2013;77(6):1136-1150

48. Fenske $\mathrm{S}$, et al. Comprehensive multilevel in vivo and in vitro analysis of heart rate fluctuations in mice by ECG telemetry and electrophysiology. Nat Protoc. 2016;11(1):61-86.

49. Fenske S, et al. Sick sinus syndrome in HCN1-deficient mice. Circulation. 2013;128(24):2585-2594.

50. McCormick DA, Bal T. Sensory gating mechanisms of the thalamus. Curr Opin Neurobiol. 1994;4(4):550-556.

51. Budde T, et al. Reciprocal modulation of I (h) and I (TASK) in thalamocortical relay neurons by halothane. Pflugers Arch. 2008;456(6):1061-1073.

52. Bal T, McCormick DA. What stops synchronized thalamocortical oscillations? Neuron. 1996;17(2):297-308

53. Vassalli A, Franken P. Hypocretin (orexin) is critical in sustaining theta/gamma-rich waking behaviors that drive sleep need. Proc Natl Acad Sci USA. 2017;114(27):E5464-E5473.

54. Bunting M, Bernstein KE, Greer JM, Capecchi MR, Thomas KR. Targeting genes for self-excision in the germ line. Genes Dev. 1999;13(12):1524-1528.

55. Hammelmann V, Zong X, Hofmann F, Michalakis S, Biel M. The cGMP-dependent protein kinase II is an inhibitory modulator of the hyperpolarization-activated HCN2 channel. PLoS ONE. 2011;6(2):e17078.

56. Schneider CA, Rasband WS, Eliceiri KW. NIH Image to ImageJ: 25 years of image analysis. Nat Methods. 2012;9(7):671-675.

57. Beaudoin GM, et al. Culturing pyramidal neurons from the early postnatal mouse hippocampus and cortex. Nat Protoc. 2012;7(9):1741-1754

58. Much B, et al. Role of subunit heteromerization and N-linked glycosylation in the formation of functional hyperpolarization-activated cyclic nucleotide-gated channels. J Biol Chem. 2003;278(44):43781-43786.

59. Koch S, et al. Gene therapy restores vision and delays degeneration in the CNGB1(-/-) mouse model of retinitis pigmentosa. Hum Mol Genet. 2012;21(20):4486-4496.

60. Treviño M. Stimulus similarity determines the prevalence of behavioral laterality in a visual discrimination task for mice. $S c i$ Rep. 2014;4:7569.

61. Treviño M, Oviedo T, Jendritza P, Li SB, Köhr G, De Marco RJ. Controlled variations in stimulus similarity during learning determine visual discrimination capacity in freely moving mice. Sci Rep. 2013;3:1048.

62. Morris R. Developments of a water-maze procedure for studying spatial learning in the rat. J Neurosci Methods. 1984;11(1):47-60.

63. Barry PH. JPCalc, a software package for calculating liquid junction potential corrections in patch-clamp, intracellular, epithelial and bilayer measurements and for correcting junction potential measurements. J Neurosci Methods. 1994;51(1):107-116.

64. Pillai AG, Anilkumar S, Chattarji S. The same antidepressant elicits contrasting patterns of synaptic changes in the amygdala vs hippocampus. Neuropsychopharmacology. 2012;37(12):2702-2711.

65. Cao-Ehlker X, et al. Up-regulation of hyperpolarization-activated cyclic nucleotide-gated channel 3 (HCN3) by specific interaction with $\mathrm{K}^{+}$channel tetramerization domain-containing protein 3 (KCTD3). J Biol Chem. 2013;288(11):7580-7589.

66. Stosiek C, Garaschuk O, Holthoff K, Konnerth A. In vivo two-photon calcium imaging of neuronal networks. Proc Natl Acad Sci USA. 2003;100(12):7319-7324.

67. Jia H, Rochefort NL, Chen X, Konnerth A. In vivo two-photon imaging of sensory-evoked dendritic calcium signals in cortical neurons. Nat Protoc. 2011;6(1):28-35.

68. D'Arcangelo G, D'Antuono M, Biagini G, Warren R, Tancredi V, Avoli M. Thalamocortical oscillations in a genetic model of absence seizures. Eur J Neurosci. 2002;16(12):2383-2393. 